\title{
Tracking and Characterization of Aircraft Wakes using Acoustic and Lidar Measurements
}

\author{
Earl R. Booth, Jr. ${ }^{*}$ and William M. Humphreys, Jr. ${ }^{\dagger}$ \\ NASA Langley Research Center, Hampton, VA, 2368-2199
}

\begin{abstract}
Data from the 2003 Denver International Airport Wake Acoustics Test are further examined to discern spectral content of aircraft wake signatures, and to compare three dimensional wake tracking from acoustic data to wake tracking data obtained through use of continuous wave and pulsed lidar. Wake tracking data derived from acoustic array data agree well with both continuous wave and pulsed lidar in the horizontal plane, but less well with pulsed lidar in the vertical direction. Results from this study show that the spectral distribution of acoustic energy in a wake signature varies greatly with aircraft type.
\end{abstract}

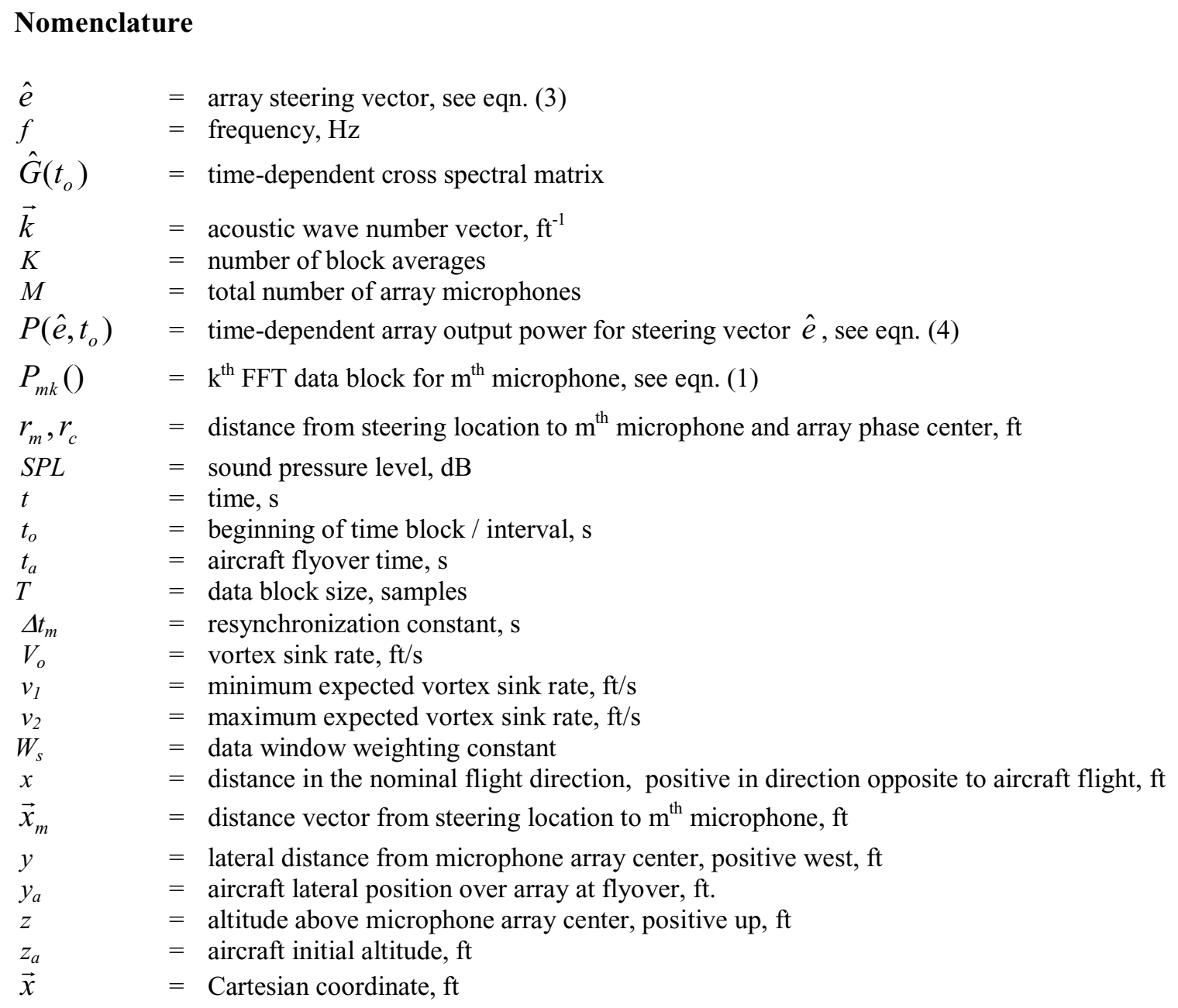

\footnotetext{
${ }^{*}$ Senior Research Engineer, Aeroacoustics Branch, Mail Stop 461.

${ }^{\dagger}$ Senior Research Engineer, Aeroacoustics Branch, Mail Stop 461, AIAA senior member.
} 


\section{Introduction}

$\mathrm{T}$ HE study of aircraft wakes using acoustic sensors, or wake acoustics, is a relatively new field of study. In fact, only a few years ago, it was considered unlikely that aircraft wakes out of ground effect made noise. It was considered to be much less likely that even if there were wake noise, that it could be studied due to the proximity to the aircraft, which surely makes much more noise. But, as Arthur C. Clarke once observed "If an elderly but distinguished scientist says that something is possible he is almost certainly right, but if he says that it is impossible he is very probably wrong."

In Europe, since the mid-1990's, DLR has developed phased microphone arrays as a field measurement tool for use in aircraft flight tests, originally to measure airframe noise. Michel ${ }^{2}$ reported that an acoustic array could be used to separate airframe and jet noise sources using a microphone array to examine noise from a military aircraft flying by the array. This work was extended ${ }^{3}$ to localize airframe noise sources on commercial aircraft during flyover. Another test campaign examined airframe noise sources on an Airbus A340 and buzz-saw noise from an Airbus A3195.

As first reported in 2002, Michel ${ }^{6}$ and his colleagues turned their array to a new sound source, the aircraft wake. Under the sponsorship of the C-WAKE program, DLR explored the feasibility of using an acoustic array to measure the vortex wake. Wake tracking in a horizontal plane, for a series of time steps, was presented for an Airbus A320, a Boeing 737, and a Boeing 757. Spectral content data for the tracked vortex wakes were presented.

Meanwhile, in the late-1990's the U.S. National Airspace System (NAS) was becoming seriously over-crowded. Aircraft delays during summer peak travel periods were wide-spread. Consequently the FAA and NASA have ongoing research programs to explore ways to expand airport capacity. Among the factors limiting airport capacity is the number of aircraft that can land on, or take off from, a given runway in a given amount of time. One factor determining maximum runway utilization is the requirement to maintain a minimum separation distance between aircraft to avoid wake encounters. Current guidelines are based on minimum separation distances determined by aircraft types for both the leading and trailing aircraft. Those rules are based on atmospheric conditions that favor longevity of the aircraft wake, since that is the most conservative case. There are some weather conditions that act to disperse the wake sooner, or to displace the wake from the path of the following aircraft, so these guidelines were examined by NASA to determine if there was a practical way to safely reduce the minimum aircraft spacing, at least under some conditions, and thereby increase airport throughput. A combination sensor- and analytical-based system, the Aircraft Vortex Spacing System (AVOSS) ${ }^{7}$, was demonstrated, in shadow-mode, at the Dallas Fort Worth International Airport in June 2000, and results indicated that operation of such a system would result in an average six percent increase in capacity compared to current practice. A proposed concept of operations for a Wake Vortex Advisory System ${ }^{8}$ (WakeVAS) has been developed under sponsorship of NASA's Airspace Systems Program. Both AVOSS and WakeVAS rely primarily on lidar sensors for wake tracking.

In the private sector, a technology derived from work done for the Department of Defense for underwater applications was proposed for application to aircraft wake detection and tracking. Flight Safety Technologies, Inc. (FST), in partnership with Lockheed Martin, developed SOCRATES ${ }^{9}$ (Sensor for Optically Characterizing Ringeddy Atmospheric Turbulence Emanating Sound), a laser-based acoustic sensor designed to detect the presence of airborne aircraft wakes from the hypothesized acoustic signature of an aircraft wake. Starting in 1997, Congress directed NASA and the Department of Transportation (DOT) to evaluate SOCRATES as a wake detection and tracking sensor. In 1998 and 2000, the FAA and NASA conducted evaluation tests of the SOCRATES system at JFK airport, using normal aircraft traffic, and at Langley Air Force Base, using the NASA Langley Boeing 757 aircraft. In spite of the success claimed by FST and Lockheed, the NASA tests showed nothing, most likely because only conventional single-microphone measurements were made. In a report ${ }^{10}$ to summarize the findings of those two tests, Volpe made the following recommendations:

"Recommendation 1: Any further development of SOCRATES sensor technology and other candidate systems dependent upon the hypothesized wake vortex sound-generation phenomenon should be deferred until such time as there is a strong, well-established phenomenological basis for their further development.

Recommendation 2: If there is any interest by the government in pursuing phenomenological research; that is, determining whether wake vortices emit unique, consistent acoustic signatures, for possible application to wake vortex detection, the investigation should be initiated and conducted prior to the development of any potential passive acoustic wake vortex sensor.

Recommendation 3: The FAA and NASA should cooperatively develop a set of requirements for an operational wake vortex sensor to support the development and deployment of a wake turbulence system for mitigation of wake turbulence constraints on airport capacity."

These recommendations were used to formulate a NASA/DOT research plan that would definitively answer the outstanding questions concerning the viability of using acoustic measurements to track aircraft wakes. The resulting 
plan and subsequent research project were formulated around three questions that closely followed the three recommendations in the Volpe report:

1. Do aircraft wakes make a sound?

2. Can the wake sound be uniquely characterized and linked to aircraft class or wake characteristic that corresponds to the strength and/or trajectory of the aircraft wake?

3. Can this wake acoustic signature be detected and tracked robustly enough to be used in a system to reliably avoid wake encounters?

In 2003, a wake acoustic test was conducted at the Denver International Airport. A test site was established approximately 2 miles north of runway 16L, and data were acquired in August and September. One of the primary sensors deployed was a large acoustic array developed by NASA, DOT, and contractors Optinav and Microstar Laboratories. SOCRATES was deployed by FST and Lockheed. A pair of acoustic arrays were deployed by DLR under contract to FST. In addition, a continuous wave lidar was deployed by MIT Lincoln Labs, a pulsed lidar by CTI, and a meteorological tower and sodar by DOT Volpe. Preliminary results from the Denver test were reported by Dougherty, et $\mathrm{al}^{11}$. In that study, time-based beamforming using decimated ${ }^{\ddagger}$ data over a frequency range of $10-400 \mathrm{~Hz}$ was used to detect, track, and analyze aircraft wake data from Boeing 757 and 737 aircraft. Horizontal tracking was used to examine wake features, including an example of Crowe instability. Vertical wake tracking data was used to compare to a theoretical model of vortex sink rates and showed good agreement. One of the questions raised in the Dougherty paper was the effect of microphone packaging on frequency response. In addition, since time-domain beamforming was used for that study, the spectral content of the wake vortices was not discernable.

In addition, several analytical studies have been conducted to characterize the aircraft wake acoustic signature. Zhang $^{12}$ examined fundamental mechanisms whereby vortices in an aircraft wake may create noise during vortex roll-up, and predicted that the acoustic energy produced by the vortex wake would exist in a frequency range below $100 \mathrm{~Hz}$. Hardin ${ }^{13}$ examined the spectral content of noise created by an aircraft wake due to several proposed mechanisms and predicted the sound would exist in frequency ranges from infrasonic to about $70 \mathrm{~Hz}$. In another study, Hardin ${ }^{14}$ predicted the acoustic signature of an aircraft wake interacting with the ground plane to be infrasonic. All of the analytical studies predicted that acoustic signatures of aircraft wakes from the fundamental mechanisms examined would exist from the infrasonic range up to about $100 \mathrm{~Hz}$. To date, measurements ${ }^{6}$ of the spectral content of aircraft wake sound has shown peak acoustic energy at frequencies much higher (on the order of $200 \mathrm{~Hz}$ ) than those predicted in the analytical studies.

\section{Description of test, coordinate system, microphone packaging, and frequency range of interest}

The present investigation extends the previous study ${ }^{11}$ to examine the spectral content of aircraft wakes for several representative aircraft types, examine the effect of sensor directivity on detection of the aircraft wake, and compare microphone phased array-derived wake tracking to tracking data provided by two types of lidar. This section addresses definition of the coordinate system, the relative placement of the sensors, the origin of the lidar data used for the tracking comparisons and the suitability of microphone packaging, and selection of the frequency range for this study.

The test site was arranged so that the acoustic array was positioned on the extended centerline of runway 16L, as shown in Figure 1. The origin of the reference coordinate system was located at the center of the acoustic array, with $x$ defined as positive north, so the aircraft were flying in the negative $x$ direction. Since $z$ is defined as positive up, $y$ is chosen to be positive west, to form a right-handed coordinate system. The continuous wave lidar ${ }^{15}$ was located adjacent to the acoustic array, approximately $260 \mathrm{ft}$ in the negative $y$ -

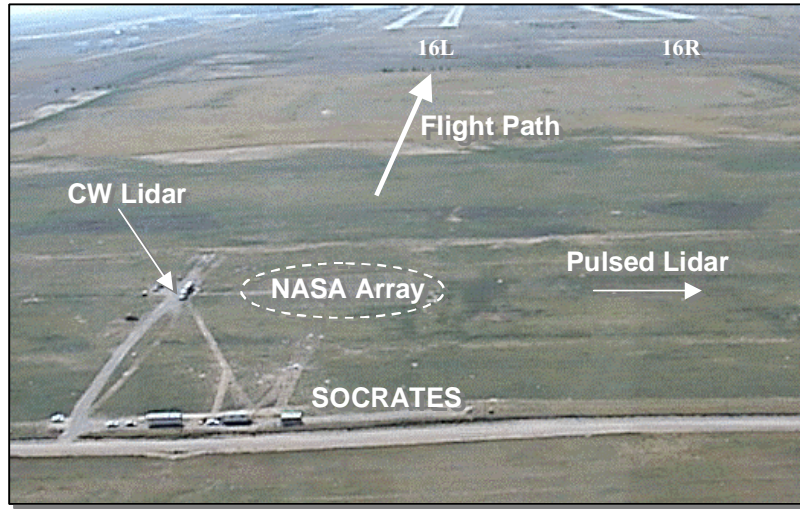

Figure 1. Test site at Denver International Airport.

\footnotetext{
\$ Data decimation is the process by which digital data are first digitally low-pass filtered at a new desired antialiasing frequency and then down sampled to reduce the number of data samples. Preliminary results report in reference 11 used data that had been decimated from 25,600 samples/s to $1024 \mathrm{samples} / \mathrm{s}$. Resulting data contained spectral information up to a maximum of about $400 \mathrm{~Hz}$. See reference 11 for further details.
} 


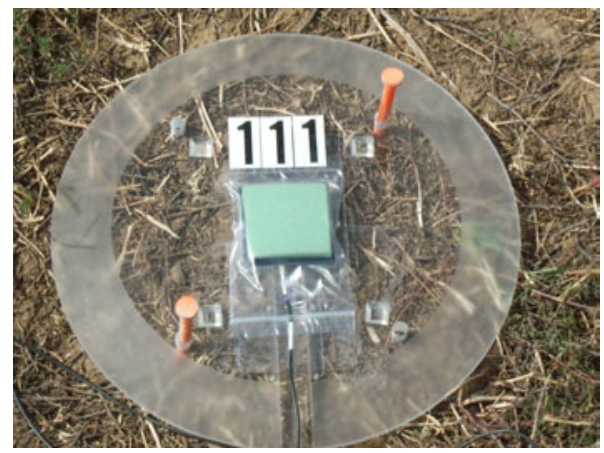

Figure 2. Microphone packaging used to waterproof microphones during test. direction. The pulsed lidar ${ }^{16}$ was located $2973 \mathrm{ft}$ in the positive $y$ direction from the center of the array. Data processed by the respective lidar operators were delivered to the Government in final data reports and those processed data are used in this study.

The Denver wake acoustics test was designed to measure vortex wakes from aircraft in final approach to the airport. An implicit assumption is that data from one flyover by a given type of aircraft should correspond to the wake from another flyover by that same type of aircraft. While that assumption has held up during the data analysis, it is important to point out that commercial aircraft land with a fixed aerodynamic configuration specified by procedure: landing flap setting, gear down, etc., and course corrections are accomplished usually by engine power adjustments Assuming the aircraft are approximately the same weight, the aircraft should be flying about the same speed for the given approach path. Examination of flyover speeds for a given aircraft type shows only a small variation, so this assumption seems reasonable. All of these data were acquired under visual flight operations, so there should be no major wake data variation effects due to weather. Hence, wake data from one flyover of a given type of aircraft should correspond to wake data from a flyover of a different aircraft of the same type. A limited number of repeat flyovers are shown in this study, although many more flyovers have been examined in the data analysis process, and these data support the assumption of similarity of wake data from different aircraft of the same type.

One of the open questions concerning the acoustic array in the previous study was the effect of microphone packaging on the performance of the microphone. During the test deployment, it was discovered that the microphone mounting system failed to keep the microphones adequately dry. The situation was resolved by packaging the microphones in a plastic bag, as shown in Figure 2. While the bag successfully kept the microphones dry for the duration of the test, the effect of the bag on the microphone frequency response was unknown. This issue was declared to be beyond the scope of the previous study, but needed to be examined for this study. Accordingly, several representative microphones selected from those used in the Denver test were calibrated in a reverberation room at NASA Langley Research Center. The calibrations compared the frequency response of the Panasonic type WM 61-A electret microphone to a quarter-inch $\mathrm{B} \& \mathrm{~K}$ model 4136 microphone and were designed to measure the effect of the microphone packaging on electret microphone frequency response. The effect of the microphone packaging is shown in Figure 3. Below a frequency of around $1.5 \mathrm{kHz}$, there is essentially no difference in microphone response due to the packaging. Between $1.5 \mathrm{kHz}$ and $5 \mathrm{kHz}$, there is a reduction in microphone sensitivity that reaches a maximum of about $10 \mathrm{~dB}$ between 2 and $3 \mathrm{kHz}$. Above $5 \mathrm{kHz}$, there is only a small reduction of

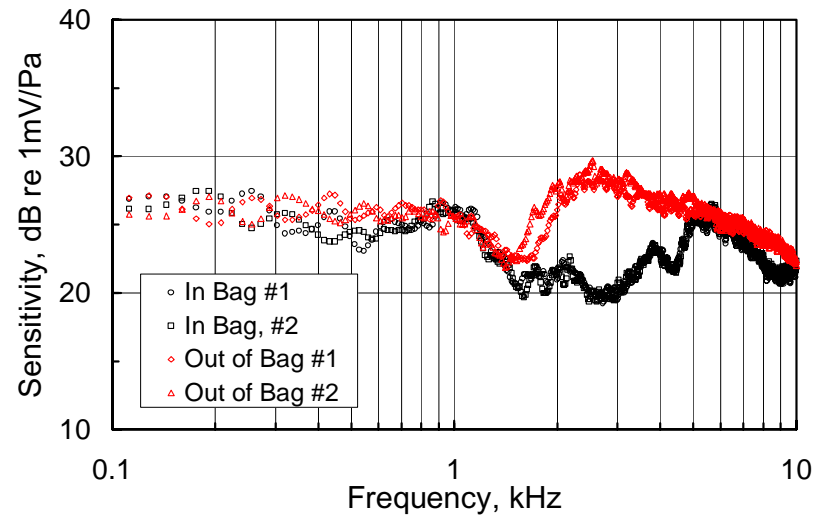

Figure 3. Packaging effect on frequency response.

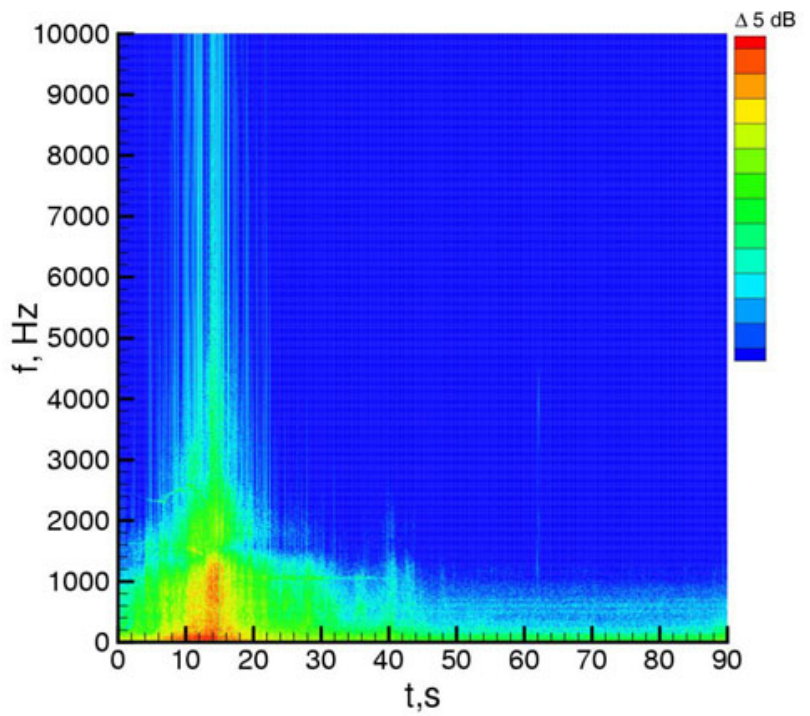

Figure 4. Spectrogram of a Boeing 737 flyover.

4

American Institute of Aeronautics and Astronautics 
microphone sensitivity. For the results reported previously ${ }^{11}$, with decimated data containing the energy up to 400 $\mathrm{Hz}$, the microphone packaging had no effect. The current study examines data in frequency ranges extending through the $500 \mathrm{~Hz}$ one third octave band, so microphone packaging had no effect on data used in this study.

While the microphone packaging has been shown to be adequate for wake acoustic testing, use of the array for other noise sources, such as aircraft airframe noise, will require packaging that will keep the microphones dry and operational with only minimal effects on microphone sensitivity. Development of improved packaging is part of a continuing effort to further improve the array system.

The frequency range of interest was determined by examination of data from individual microphones processed into spectrograms for the duration of the data record. An example is the spectrogram in figure 4. Data were acquired up to a frequency of $10 \mathrm{kHz}$ in the Denver test. In Figure 4, it is evident that except for the aircraft flyover at about 12 seconds, the spectral energy is mostly contained between 0 and $2 \mathrm{kHz}$. Ten to forty seconds after the aircraft flyover, the majority of the acoustic energy exists at frequencies less than $1 \mathrm{kHz}$. This study will examine the one third octave frequency bands between 100 and $500 \mathrm{~Hz}$, because, as will be shown subsequently, most of the wake acoustic energy exists in the $100-$ to $315-\mathrm{Hz}$ one third octave bands.

\section{Analysis of Acoustic Data}

Data from the test were examined to characterize the spectral content for several types of aircraft using beamforming to track the wake in a vertical plane at specific times. These data were further processed to generate wake trajectories in $\mathrm{y}$ and $\mathrm{z}$ as a function of time. Data beamformed in horizontal planes were used to map the lateral position of the wake as a function of $\mathrm{x}$ at a given time $\mathrm{t}$. These data were further processed to obtain the wake lateral distribution as functions of $\mathrm{y}$ and $\mathrm{t}$. This section outlines the methods used to analyze the acoustic data in the present study.

\section{A. Beamforming}

A frequency-domain beamforming algorithm was developed for the present study which allowed extraction of time-dependent spectral information from the acquired array data. This choice of analysis differs from previous time-domain algorithms which have been applied to wake vortex data. ${ }^{11,17}$ The previous algorithms operated on decimated data and were designed to maximize the localization capability of the array at the expense of lost spectral information. The algorithm employed for this study, while computationally more expensive, preserved spectral content in the beamforming output.

Post processing of acquired data from the array microphones started with computation of the time-dependent cross-spectral matrix for each examined flyover. The Fast Fourier Transform (FFT) was used to compute each element of the matrix in the data ensemble. After converting the raw data to engineering units, the transform pairs $P_{m}\left(f, t_{o}, T\right)$ and $P_{m},\left(f, t_{o}, T\right)$ were formed from time records $p_{m}(t)$ and $p_{m},(t)$, respectively. The discrete time records were defined for a time spacing of $\Delta t$, starting at time $t_{o}$ and extending over a data block size $T$ for microphones $m$ and $m^{\prime}$. The individual cross spectrum matrix element for starting time $t_{o}$ was computed via

$$
G_{m m^{\prime}}\left(f, t_{o}\right)=\frac{2}{K W_{s} T} \sum_{k=1}^{K}\left[P_{m k}^{*}\left(f, t_{o}-\Delta t_{m}, T\right) P_{m^{\prime} k}\left(f, t_{o}-\Delta t_{m^{\prime}}, T\right)\right]
$$

where $K$ is the number of block averages, $W_{s}$ is the data window weighting constant (a Hamming window was typically used), and the total record length is $T_{t o t}=K T$. The terms $\Delta t_{m}$ and $\Delta t_{m}$, represent empirically determined resynchronization constants to correct for random time shifts in the data acquired from the various 8-channel data acquisition boards comprising the hardware system. The technique for determining these empirical constants was developed by Dougherty and is described in reference 11. For the present study, the data block size was defined as 8192 samples and four block averages were performed, yielding a total record length of 1.28 seconds. While such a record length would be excessive for measurements of aircraft noise (due to smearing of the sources during aircraft passage), this record length is reasonable for measurement of wake vortices which have a much smaller lateral velocity and sink rate with respect to the array.

Using equation (1), the full matrix, with $M$ being the total number of microphones in the array, was formed as 


$$
\hat{G}\left(t_{o}\right)=\left[\begin{array}{cccc}
G_{11}\left(t_{o}\right) & G_{12}\left(t_{o}\right) & \cdots & G_{1 M}\left(t_{o}\right) \\
& G_{22}\left(t_{o}\right) & & \vdots \\
& & \ddots & \vdots \\
& & & G_{M M}\left(t_{o}\right)
\end{array}\right]
$$

where the dependency on $f$ has been dropped from the notation for convenience. The lower triangular elements of this Hermitian matrix are complex conjugates of the upper triangular elements. Note that all cross spectral matrix elements were employed in subsequent processing, with no modification of the diagonal terms.

A classical delay and sum beamforming approach was used for analysis of the array data. This approach assumed monopole distributions of measured wake acoustic sources. A scanning plane of grid points was first defined over the noise source region of interest. The array was then electronically "steered" to each defined grid point in sequence. For each grid location, a steering vector $\hat{e}$ containing the retarded-time phase adjustment for each microphone in the array was defined as

$$
\hat{e}=\left[\begin{array}{c}
\frac{r_{1}}{r_{c}} \exp \left[j\left(\vec{k} \cdot \vec{x}_{1}\right)\right] \\
\vdots \\
\frac{r_{m}}{r_{c}} \exp \left[j\left(\vec{k} \cdot \vec{x}_{m}\right)\right]
\end{array}\right]
$$

where $\dot{k}$ is the acoustic wave vector, $x_{m}$ is the distance vector from the steering location to each microphone $m$, and the ratio $\left(r_{m} / r_{c}\right)$ is included to normalize the relative position vector related amplitude to that of the phase center of the array. The time dependent output power of the array at the steering location was obtained from

$$
P\left(\hat{e}, t_{o}\right)=\frac{\hat{e}^{T} \hat{G}\left(t_{o}\right) \hat{e}}{M^{2}}
$$

where the subscript $T$ denotes a complex transpose of the vector, and $P\left(\hat{e}, t_{o}\right)$ is the mean-squared pressure at time $t_{o}$. The division by $M^{2}$ serves to reference the array output to an equivalent single microphone output level. Equation (4) represents the steered output power of the array for a single frequency. For processing performed for this study, one third octave bands were desired for horizontal plane processing and a broadband frequency content was desired for vertical plane processing. The pressure-squared values of the array output power were summed over the desired band for each type of processing. Note that spectral band levels were calculated after completion of the vectorial (complex) operations depicted in equation (4). This prevented possible significant bias errors in summing across phase-shifted cross spectral bands. Reference 18 contains more details regarding the general use of the classical beamforming algorithm which was employed for this study.

\section{B. Vortex Trajectory Processing}

In order to determine the aircraft flyover time, aircraft initial altitude and vortex sink rate $V_{o}$, a vertical scanning plane was defined which bisected the array perpendicular to the flight direction. The vertical plane extended in altitude from 300 to 1000 feet, and in the transverse direction from -300 to +300 feet, using a grid resolution of 4 feet. The first 40 seconds of each flyover ensemble time series was broken up into individual one second intervals, and equations (1) through (4) were applied to each time interval and each grid point to generate a set of vertical plane measurements, each associated with a given time step. The aircraft flyover time was determined by selecting the vertical plane containing the peak mean-squared power for the entire data set. The peak power was taken to be the aircraft passage through the vertical beamforming plane. The time of aircraft passage, $t_{a}$, was the time associated with the peak power, and the location of this peak power yielded the $y_{a}$ and $z_{a}$ coordinates. 
Subsequent vortex wake trajectory locations were determined for the remaining time steps by selecting the maximum mean-squared power in a window defined by the initial aircraft location. Limits for that window are given by

$$
\begin{gathered}
y_{a}-150 \mathrm{ft} \leq y \leq y_{a}+150 \mathrm{ft} \\
z \leq z_{a} v_{1}\left(t-t_{a}\right) \\
z \geq z_{a} v_{2}\left(t-t_{a}\right)
\end{gathered}
$$

where $v_{1}$ and $v_{2}$ are the least and greatest expected vortex descent rates, and the values of 0 . and $25 \mathrm{ft} / \mathrm{s}$ were used, respectively. If the maximum mean-squared power inside this window was greater than the magnitude of the array gain for that time step, then $y$ and $z$ coordinates of that point were added to the vortex trajectory. Since only one point per time step was selected using this method, only one of the vortices in the wake could be tracked for a given time step, and it is not assured that the same vortex will be tracked for different time steps. Vortex trajectory data were then used to estimate the actual vortex sink rate $V_{o}$.

Vortex wake trajectory data are compared with continuous wave and pulsed lidar data in the results section. It is known that the microphone array has much better horizontal resolution than vertical, so it is expected that the vortex wake will be tracked better horizontally than vertically.

\section{Lateral Distribution of Vortex Wake Processing}

Upon the completion of the vertical plane processing, a series of horizontal scanning planes were defined and centered above the array. The altitude of the first scanning plane corresponded with the aircraft initial altitude ascertained from the vertical processing. Subsequent horizontal plane altitudes were computed using

$$
\begin{array}{cc}
z=z_{a} & \text { when } t_{o}<t_{a} \\
z=z_{a}-V_{o}\left(t_{o}-t_{a}\right) & \text { when } t_{o} \geq t_{a}
\end{array}
$$

where $z_{a}$ is the aircraft initial altitude and $t_{a}$ is the aircraft flyover time. In other words, the horizontal plane altitude was held constant from the start of the time record until just after aircraft passage at which point the plane descended at the vortex sink rate. Each horizontal plane extended in the flyover direction from -300 to +300 feet and in the transverse direction from -300 to +300 feet, using a grid resolution of 4 feet. As with the vertical processing, the array ensemble time series was broken up into one second intervals, equation (6) was used to determine the horizontal plane altitude, and then equations (1) through (4) were applied to each time interval and grid location to map the distribution of wake vortex noise as a function of time.

These distributions were depicted as individual color contour plots of the sound pressure level as a function of location for each one-second time interval. Animations of the contour plots allowed the time evolution of the wake location and strength to be examined. However, the individual time step frames resulted in rather unwieldy data sets, creating the desire to examine the entire wake vortex evolution in a single frame.

One way to accomplish this goal is to select all the data on the $x=0$. line of each frame and then plot that as a function of $y$ and $t$. Another way to accomplish this is to select all the points inside an aperture of a range in the $\mathrm{x}$ - direction and integrate over that range to provide an SPL vs. y curve for each t, which provides an enhancement of the vortex signal at each time step. Variation of the aperture parameter also provides a means to examine effects of

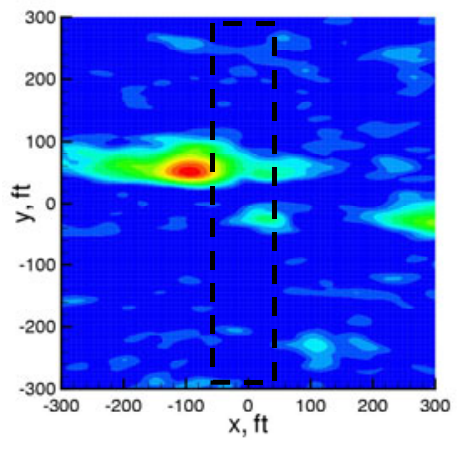

a) Horizontal frame $x-y$ plot for $t=20 s$.

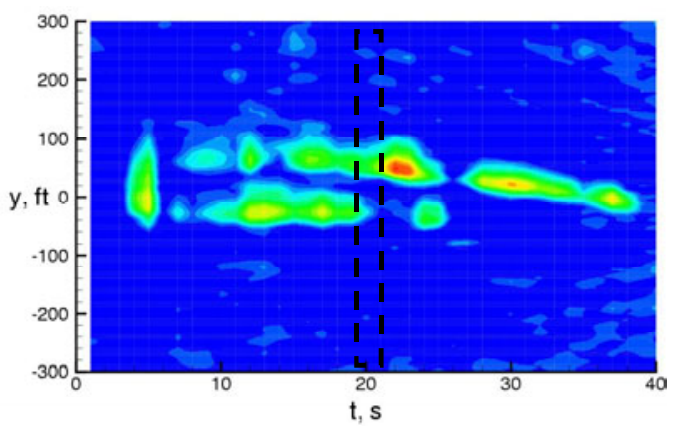

b) Lateral time history plot.

Figure 5. Lateral wake time history from individual frame time step data. 
sensor directivity on detectability of wake signals, at the expense of some smearing of lateral vortex coordinates, especially for those cases affected by crosswinds. The lateral time history is constructed from the integrated sound power in the selected aperture for each time step. As shown in Figure 5, the energy in an aperture of $+/-50 \mathrm{ft}$ in the $\mathrm{x}$-direction (as indicated by the dashed lines in part a) is integrated in the x-direction and added to the lateral time history plot (as indicated by the dashed lines in part b). Lateral time history plots were constructed for each aircraft flyover for each of the one third octave bands between 100 and $500 \mathrm{~Hz}$. It is interesting to note that since the aircraft were flying at about $240 \mathrm{ft} / \mathrm{s}$ over the array, a 40 second record is equivalent to a vortex age of 64 wingspans for an aircraft with a $150 \mathrm{ft}$ wingspan. As a result, to be scaled proportionally in the time direction, the plot shown in Figure $5 \mathrm{~b}$ would need to be stretched about 25 times in the time direction, which would result in a much more linear vortex track than shown in the figure.

\section{Results}

\section{A. Comparison of Acoustically-derived Tracking with Lidar Data}

Vortex trajectory data are presented for a number of different aircraft classes in Figures 6-18. As a matter of convenience, the aircraft are grouped both by type and by manufacturer, with Boeing aircraft first, followed by McDonnell Douglas, Airbus, and Bombardier. Aircraft flyover time is subtracted from the acoustic data record time for comparison with lidar data. In addition, a grayscale contour plot corresponding to the $100 \mathrm{~Hz}$ one third octave band spectral content plot is added to the lateral tracking plot. Since that data is derived from a horizontal plane beamforming of the acoustic data and the tracking data is derived from vertical beamforming of the same data, this provides a good cross-check of acoustic results. Finally, the particular data file number is included in all the data figures for reference. A typical file number, such as 030915_130907 was obtained on September 15, 2003 at 1:09:07 pm local Mountain Daylight Savings time.

In Figure 6, a set of tracking data is presented for a Boeing 737-300 aircraft. Vortex lateral tracking looks reasonable until approximately 26 seconds after the aircraft passage. Limited lidar data available for this flyover tends to agree with lateral wake placement, but scarcity of lidar data makes this case less than conclusive. Altitude data derived from the microphone array tends to indicate a range of wake descent speeds. The limited lidar data also indicates that some portion of the wake may be descending faster than others. The continuous wave lidar data also indicates that the initial aircraft altitude is about $600 \mathrm{ft}$, while the microphone array indicates a 700 - $\mathrm{ft}$ altitude. The 700-ft altitude is more consistent with the lidar data. In Figure 7, data from another Boeing 737-300 once again shows good agreement with lateral wake placement, until approximately 22 seconds after aircraft passage. In this case, there is enough lidar data to make a reasonable comparison. It is interesting that while the 737 has a wingspan of $94 \mathrm{ft} 9$ in, the spacing between the wake vortices, based on the lidar data, seems to be between 50 and $100 \mathrm{ft}$. In this case, the microphone array altitude data exhibits a wide scatter, and only the upper bound of the altitude data corresponds at all with lidar data. Interestingly, pulsed lidar indicates that the wake altitude is decreasing faster than the $\mathrm{cw}$ lidar data. For the Boeing 737-300 cases, the lateral time history corresponds well with lidar data, but altitude data corresponds less well. The pulsed lidar data seems to indicate a faster wake descent than the cw lidar for the second aircraft. Both sets of lidar data indicate for the second aircraft that vortex lateral separation varies between one-half to one aircraft wing span.

In Figure 8, tracking data for a Boeing 757-200 shows excellent agreement between lidar and acoustic data for lateral tracking. The vortex separation distance is between 150 and $100 \mathrm{ft}$, which corresponds to one to two-thirds of a wingspan. Once again, vertical tracking data obtained from the microphone array is scattered, but in this case the scatter is just enough to encompass both the $\mathrm{cw}$ and pulsed lidar data. The vertical tracking data from the microphone array seems to be good to approximately 22 seconds after aircraft passage, although the lateral tracking data appears reasonable at that time. In Figure 9, for another Boeing 757-200 flyover, the lateral tracking shows excellent agreement with the lidar data. From the lidar data, the wake vortex separation is even less than that shown in Figure 8. Vertical tracking data is once again scattered, however the scatter encompasses both sets of lidar data. As before, the vertical tracking data is good until 22 seconds after aircraft passage.

In Figure 10, tracking data for a Boeing 767-300 shows less agreement in the lateral direction than was evident for either the 737 or 757 cases. Vertical tracking is much worse, with microphone array data indicating a wake altitude much lower than either the $\mathrm{cw}$ or pulsed lidar. Note that the largest member in this subset of Boeing aircraft is more difficult to track than either of the other Boeing aircraft.

Data for three versions of the McDonnell Douglas MD-80-class aircraft are presented in Figures 11-13. In Figure 11, tracking data for an MD-81 is presented. Lateral tracking is acceptable until 20 seconds after aircraft passage. Vortex separation in this case is shown to be between about 50 and $100 \mathrm{ft}$, or between a one-half and one 
full wingspan. Vertical tracking data shows that the microphone array data indicated a greater altitude than lidar data, and although there is good agreement between 10-20 seconds after aircraft passage, the overall acoustic vertical tracking for this case indicates that the wake drops much faster than is indicated by either lidar data set. Data for an MD-82, presented in Figure 12, shows good agreement with lateral tracking until 20 seconds after aircraft passage. Although the microphone data indicated an aircraft flyover height closer to the lidar data, the wake descent rate derived from the acoustic data is once again much greater than the lidar data. In Figure 13, data from an MD-87 flyover indicates that lateral tracking is acceptable until 20 seconds after aircraft passage, although there is better agreement with pulsed lidar than with $\mathrm{cw}$ lidar for this case. Vertical tracking data shows that the microphone array data indicated a lower aircraft altitude and once again indicated a faster wake descent than lidar data. So, although the microphone array lateral tracking for the McDonnell Douglas aircraft was not as good as it was for the Boeing aircraft, the microphone vertical tracking data was much worse, indicating that the vortex wake had dropped much faster than lidar data indicates. In short, vertical tracking data from the microphone array indicated that the wake had dropped out of the landing corridor when lidar data indicated that was not the case, and this false-negative measurement would not be a desirable result for a wake-avoidance sensor.

Tracking data for several Airbus A320-series aircraft are presented in Figures 14-16. In Figure 14, data for an A319 is presented. In this case the lateral tracking data illustrates a good match with both sets of lidar data. However, microphone array data indicates that the vortex wake is much lower than shown by either set of lidar data. In Figure 15, data from an A320 flyover again shows good agreement with lateral tracking and once again, vertical tracking data from the microphone array indicates the wake is lower than for either lidar data set. Tracking data from another A320 flyover shown in Figure 16 shows pretty much the same trend.

Tracking data from a Bombardier CRJ-200 flyover is presented in Figure 17. Although the CRJ is a much smaller aircraft than those mentioned to this point, the aircraft exhibits a relatively strong wake signal. Lateral tracking data for the CRJ-200 shows good agreement with lidar data until 20 seconds after aircraft passage. Vertical tracking from the microphone array once again shows the vortex wake to descend faster than lidar data.

In summary, lateral tracking data from the microphone array agrees well with lidar data until approximately 20 seconds after aircraft passage. Vertical tracking data from the microphone array, even when the aircraft passage altitude is correct, indicates that the vortex wake is at a lower altitude than lidar data. These results could be due to the relatively simple tracking algorithm used to derive the coordinates from the microphone data, the strength of the wake acoustic signal in comparison with ambient acoustic levels, relative vortex wake acoustic strength due to aircraft configuration, or very likely a combination of all three factors.

\section{B. Spectral Content of Wake Acoustic Signal for Several Aircraft Types}

Each of the aircraft flyovers examined in the previous figures was also processed using horizontal beamforming planes which were then reduced into lateral time history plots for the following one third octave bands: 100, 125, $160,200,250,315,400$, and $500 \mathrm{~Hz}$. The resulting lateral trajectory plots are presented in Figures 18-29, and correspond to the trajectory data presented in Figures 9-17. SPL levels plotted in these figures are normalized by frame gain levels from the original $x-y$ sound maps.

In Figure 18, the lateral time history for a 737-300 flyover is presented for each of the frequency bands. As was shown in Figure 7, the aircraft wake is affected by a cross wind and traverses in the y direction. In this figure, it is very apparent that the $100-$ and $125-\mathrm{Hz}$ one third octave bands are the dominant frequency bands for this flyover. Wake trajectory is also clearly delineated in the $160-$ and $200-\mathrm{Hz}$ bands, but only a small portion of the wake is apparent in the $250-\mathrm{Hz}$ band in Figure 18e. The wake is not readily apparent in the 315-, 400-, or 500-Hz band data, and even the aircraft flyover is barely discernable in the $400-$ and $500-\mathrm{Hz}$ band data. Another Boeing 737-300 flyover is presented in Figure 19 and shows the same trends with frequency band.

A flyover by a Boeing 757-200 is shown in Figure 20. Once again the wake is predominantly shown in the 100and $125-\mathrm{Hz}$ bands. In this case, the wake shows up well in the $160-$ and $200-\mathrm{Hz}$ bands, and is clearly discernable in the $250-$ and $315-\mathrm{Hz}$ bands. Neither the aircraft nor wake are discernable in the $400-$ and $500-\mathrm{Hz}$ data. Another 757-200 flyover shown in Figure 21 is similar in frequency distribution, except the aircraft and wake are discernable in the 400 and $500 \mathrm{~Hz}$ data. The wake signal of the Boeing 757 appears to be much stronger than the wake signal of the 737 .

A flyover by a Boeing 767-300 shown in Figure 22, however, shows very little wake signal. Most of what could be a wake appears in Figure 22d in the $200-\mathrm{Hz}$ band. Clearly, then, aircraft wake signals are not linearly associated with aircraft size/weight. In addition, tracking for the 767 case in Figure 10, was shown to be much worse than for the 757 or 737 cases, and perhaps this is due to the less discernable wake shown in Figure 22.

Another aircraft family, from McDonnell Douglas, is shown in Figures 23-25. An MD-81 flyover is shown in Figure 23 and shows that the wake is most apparent in the $100-$ to $160-\mathrm{Hz}$ bands. The wake is discernable in the 
$200-\mathrm{Hz}$ band, but only a small remnant of the wake signal is evident in the $250-\mathrm{Hz}$ band shown in figure $25 \mathrm{e}$. In Figure 26, the wake is not well defined until nearly 20 seconds after aircraft flyover. In this case, however, the most dominant wake signal appears in figure $24 \mathrm{c}$ near the 32 -second point in the record. Like the MD-81 flyover (Figure 23), there is little apparent wake signal in the $250-$ to $500-\mathrm{Hz}$ bands. Like the MD-82 flyover, an MD-87 flyover (Figure 25) shows a large time gap between aircraft flyover and peak wake levels. This relatively large time gap between aircraft flyover and beginning of a discernable wake vortex signal may be related to a delayed vortex rollup process unique to this particular aircraft configuration. Nationally, the dominant wake signal level appears in the $100-\mathrm{Hz}$ band for this case. While there are less evident wake signal remnants in the $125-$ to $200-\mathrm{Hz}$ bands, there is only very little remnant in the 250 - to $500-\mathrm{Hz}$ bands.

In Figure 26, a flyover by an Airbus 319 displays little if any wake signal in the $100-$ to $160-\mathrm{Hz}$ bands. While there appears to be some signal in the $200-$ to $315-\mathrm{Hz}$ bands, it is weak compared to the aircraft flyover signal. There appears to be no discernable wake in the $500-\mathrm{Hz}$ band. In Figure 27, an Airbus 320 flyover, shows a readily detectable wake in the 100 - to $200-\mathrm{Hz}$ bands, with some remnants in the remaining frequency bands. Although the wake signal only appears much later after the A320 flyover in Figure 28, the frequency content trends are similar to those shown in Figure 27.

Flyover data from a Bombardier CRJ-200 is presented in Figure 29. Interestingly, for this smaller aircraft, the primary wake frequency band is $125 \mathrm{~Hz}$. The wake is clearly discernable in the $100-\mathrm{Hz}$ and $160-$ to $315-\mathrm{Hz}$ bands. There are wake signal remnants evident in the $400-$ and $500-\mathrm{Hz}$ bands. Since this is the smallest aircraft examined in this study, it is surprising that the wake signal for this aircraft is much stronger than for the Airbus aircraft shown in figures 26-28 and the Boeing 767 shown in figure 22. The CRJ-200 aerodynamic arrangement is similar to the MD-80 series aircraft, yet the wake signal did not show the same time delay between aircraft flyover and wake signal evident in the MD aircraft case shown in figures 23-25.

\section{Conclusions}

This research project is framed by three fundamental questions:

1. Do aircraft wakes make a sound?

2. Can the wake sound be uniquely characterized and linked to aircraft class or wake characteristic that corresponds to the strength and/or trajectory of the aircraft wake?

3. Can this wake acoustic signature be detected and tracked robustly enough to be used in a system to reliably avoid wake encounters?

We know the answer to the first question: aircraft wakes make sound. It is not yet clear what generates the sound from an aircraft wake, but the amplitude and spectral content of the wake acoustic signature is dependent on the aircraft configuration. These results were known prior to this study, as reported by Michel. ${ }^{6}$

This study has been centered around addressing the second question. It is noted that wake acoustic signals were not necessarily louder, nor easier to detect, for larger, heavier aircraft than for smaller and lighter aircraft. The Boeing 767, for example, has little wake signal compared to either the Boeing 757 or 737 cases. The smallest aircraft in this study, a Bombardier CRJ-200 has a rather distinct wake signal. For data measured from aircraft near landing, these results are likely heavily influenced by the operation of the high-lift systems for the aircraft, which may help explain why aircraft of similar size exhibit different wake acoustic signal characteristics. Although the Airbus aircraft are similar in weight to the Boeing 737 and the MD-80 series aircraft, the Boeing clearly has the more detectable wake, while the Airbus aircraft wake were less easy to detect. For an aircraft with a deployed high lift system, the spanwise distribution of the lift is more inboard than for cruise conditions. The data shown here suggest that the vortex spacing may be somewhat closer than for a nominal cruise spanwise lift distribution, which is consistent with a high-lift inboard loading dominated aircraft configuration. Future analytical studies may benefit from careful consideration of spanwise lift distribution of the aircraft and details of the high-lift system.

Comparison of acoustic data with data from both pulsed and continuous wave lidar is a starting point towards addressing the third question. It is apparent that the acoustic data agree well with lidar data in the horizontal direction, but less well in the vertical direction. Those cases where the wake acoustic signal is readily detectable seem to correspond to those cases with better vertical tracking. However, in some cases, the acoustic data indicates that the wake has dropped below the approach corridor when lidar data shows that not to be the case. This falsenegative result is the least desirable result from a wake avoidance perspective. While the current results may be improved upon via improved array design and algorithm development, it is clear that lateral displacement of the wake can be fairly readily detected by the acoustic array, and the indication that the wake is laterally displaced from the descent corridor is shown to be fairly robust. 
Future work in this area may include array and algorithm development for improved measurement of vertical wake tracking, as well as further analytical work to model the effects of aircraft configuration, particularly the effects of the high-lift system, on wake acoustic signature and trajectory.

\section{Acknowledgments}

This work was sponsored by the Efficient Aircraft Spacing Project, of the NASA Airspace Systems Program. Thanks to Allan Zuckerwar and Brian Elbing, NASA Langley, for measurement of the microphone frequency response shown in figure 3. In addition, the authors acknowledge the contribution of the field measurement crew from Volpe, Titan, Optinav, and Microstar Laboratories, in particular Frank Wang, Volpe, and Robert Dougherty, Optinav.

\section{References}

${ }^{1}$ Clarke, A. C., Profiles of the Future, 1961.

${ }^{2}$ Michel, U., Barsikow, B., Haverich, B., Shuttpelz, M., "Investigation of Airframe and Jet Noise in High-Speed Flight with a Microphone Array," Proceedings of the $3^{\text {rd }}$ AIAA/CEAS Aeroacoustics Conference, AIAA, Reston, VA, pp.87-97.

${ }^{3}$ Michel, U., Barsikow, B., Helbig, J., Hellmig, M., Shuttpelz, M., "Flyover Noise Measurements on Landing Aircraft with a Microphone Array," AIAA-1998-2336, presented at the $4^{\text {th }}$ AIAA/CEAS Aeroacoustics Conference, Atlanta, GA, June 1998.

${ }^{4}$ Piet, J. F., Michel, U., Bohning, P., "Localization of the Acoustic Sources of the A340 with a Large Phased Microphone Array during Flight Tests," Proceedings of the AIAA $8^{\text {th }}$ AIAA/CEAS Aeroacoustics Conference and Exhibit, AIAA, Reston, VA, pp.1189-1199.

${ }^{5}$ Siller, H. A., Michel, U., "Buzz-Saw Noise Spectra and Directivity from Flyover Tests," Proceedings of the AIAA $8^{\text {th }}$ AIAA/CEAS Aeroacoustics Conference and Exhibit, AIAA, Reston, VA, pp.1619-1625.

${ }^{6}$ Michel, U., Bohning, P., "Investigation of Aircraft Wake Vortices with Phase Microphone Arrays," Proceedings of the AIAA $8^{\text {th }}$ AIAA/CEAS Aeroacoustics Conference and Exhibit, AIAA, Reston, VA, pp.1134-1142.

7 O'Connor, C., J., Rutishauser, D. K., "Enhanced Airport Capacity Through Safe, Dynamic Reductions in Aircraft Separation: NASA's Aircraft Vortex Spacing System (AVOSS),” NASA TM-2001-211052, August 2001.

${ }^{8}$ Rutishauser, D. K., Lohr, G., Hamilton, D., Powers, R., McKissick, B., Adams, C., Norris, E., "Wake Vortex Advisory System (WakeVAS) Concept of Operations," NASA/TM-2003-212176, April 2003.

${ }^{9}$ Cotton, W., Williams, R., Project SOCRATES: A New Sensor Technology for the Enhancement of Aviation Safety and Capacity," presented at the 2002 FAA Airport Technology Conference, Atlantic City, NJ, May 2002.

${ }^{10}$ Rudis, R. P., Wang, F., Y., Daskalakis, A. C., "Status Report SOCRATES Concept Exploration Effort,” DOT-VNTSCRSPA-01-04, October 2001.

${ }^{11}$ Dougherty, R. P., Wang, F. Y., Booth, E. R., Jr., Watts, M. E., Fenichel, N., D’Errico, R. D., "Aircraft Wake Vortex Measurements at Denver International Airport," Proceedings of the AIAA $10^{\text {th }}$ AIAA/CEAS Aeroacoustics Conference and Exhibit, AIAA, Reston, VA.

${ }^{12}$ Zhang, Y., Wang, F. Y., Hardin, J. C., “Spectral Characteristics of Wake Vortex Sound During Roll-Up,” NASA CR-2003212673, December 2003.

${ }^{13}$ Hardin, J. C., Wang, F. Y., "Sound Generation by Aircraft Wake Vortices,” NASA CR-2003-212674, December 2003.

${ }^{14}$ Hardin, J. C., Wang, F. Y., Wassaf, H., "Sound Generation by Aircraft Wake Vortices Interacting with the Ground Plane," Proceedings of the AIAA $10^{\text {th }}$ AIAA/CEAS Aeroacoustics Conference and Exhibit, AIAA, Reston, VA.

${ }^{15}$ Heinrichs, R. M., Freehart, R. E., Dasey, T. J., Mandra, R., "Development and Performance of a CW Coherent Laser Radar for Detecting Wake Vortices", Coherent Laser Radar, Vol. 19, OSA Technical Digest Series, Optical Society of America, Washington DC, 1995, pp. 186-189.

${ }^{16}$ Hannon, S. M., "Pulsed Doppler Lidar for Terminal Area Monitoring of Wind and Wake Hazards", Proceedings of the AMS 11th Conference on Aviation, Range, and Aerospace Meteorology Meeting, Hyannis, MA, October 2004.

${ }^{17}$ Dougherty, R. P., "Advanced Time-domain Beamforming Techniques", Proceedings of the AIAA 10 ${ }^{\text {th }}$ AIAA/CEAS Aeroacoustics Conference and Exhibit, AIAA, Reston, VA.

${ }^{18}$ Humphreys, Jr., W. M., Brooks, T. F., Hunter, Jr., W. W., and Meadows, K. R., "Design and Use of Microphone Directional Arrays for Aeroacoustic Measurements", proceedings of the AIAA 36th Aerospace Sciences Meeting and Exhibit, AIAA, Reston, VA. 

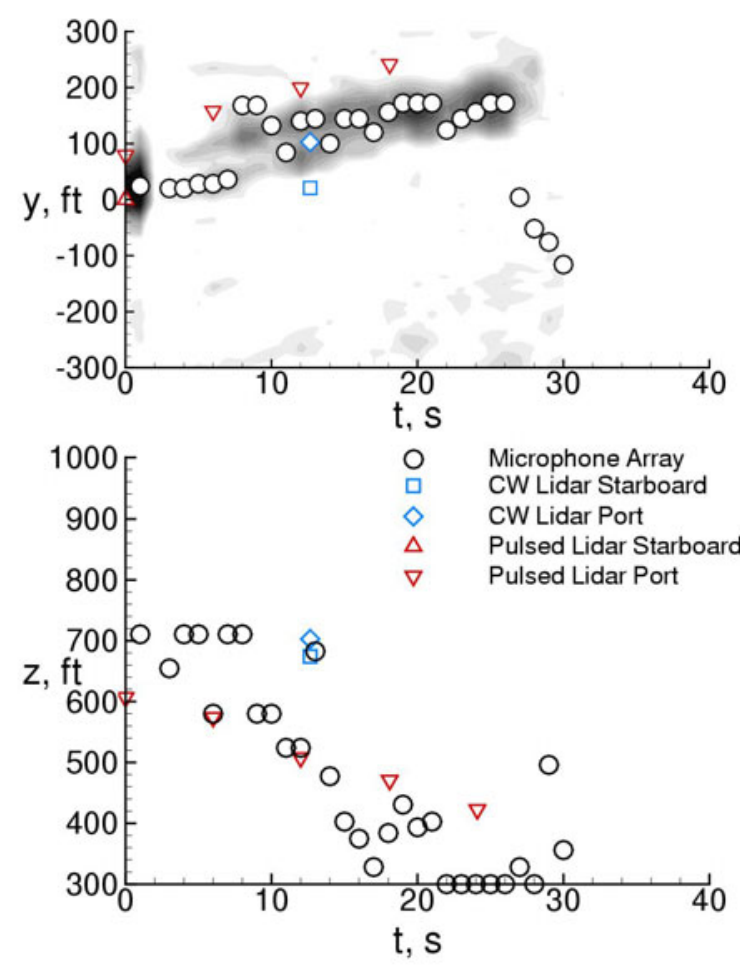

Figure 6. Tracking comparison for a Boeing 737300 (030915_130907).
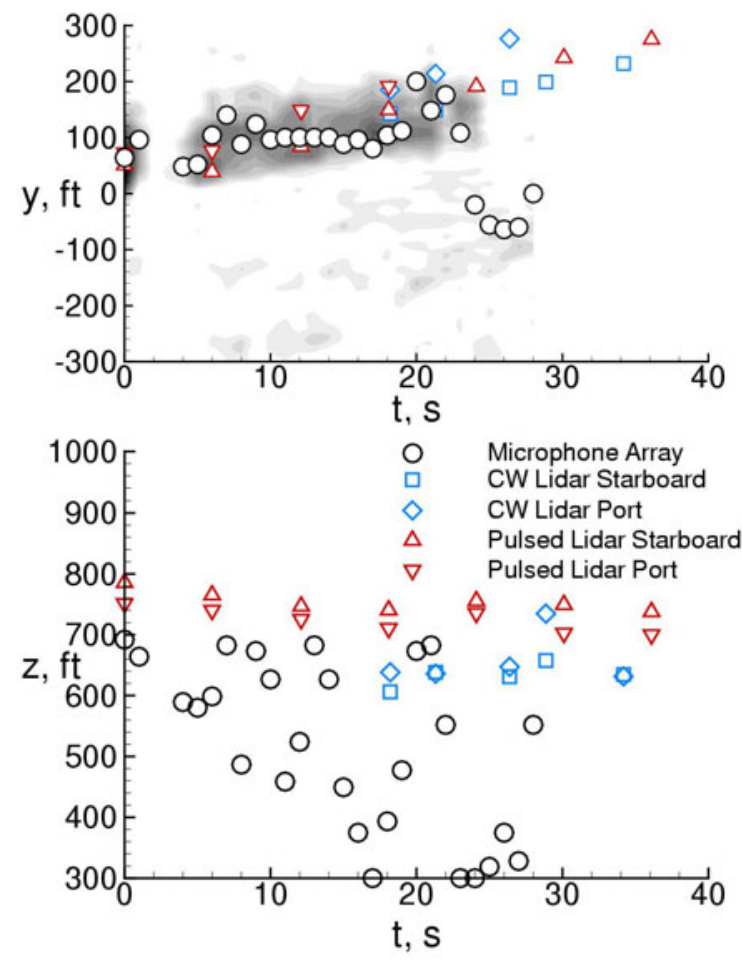

Figure 7. Tracking comparison for a Boeing 737300 (030916_104400).
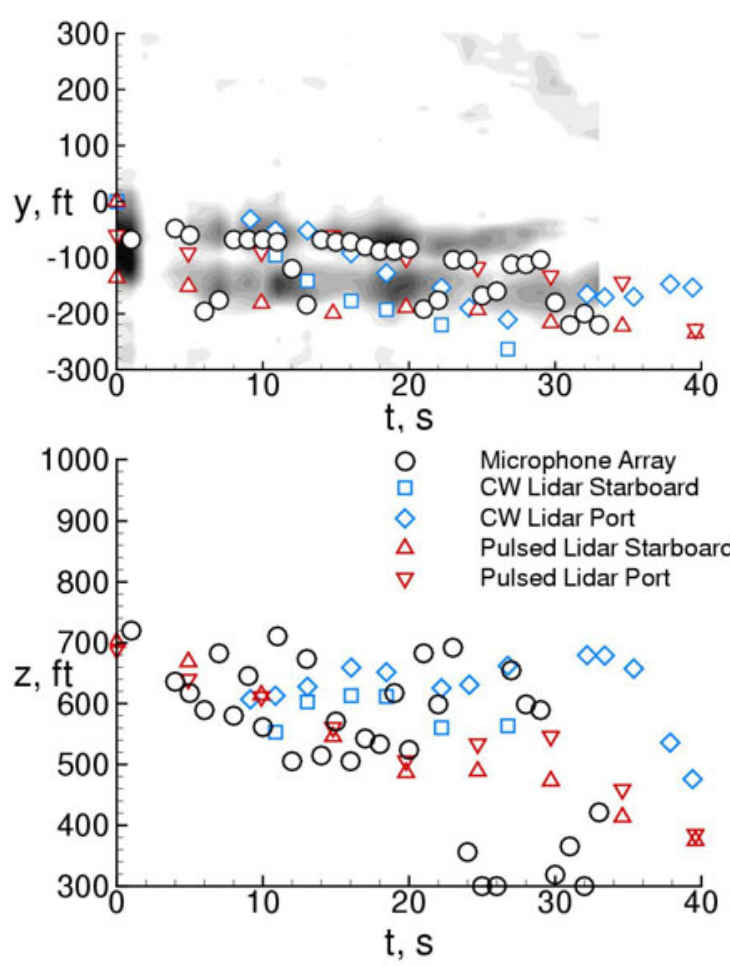

Figure 8. Tracking comparison for a Boeing 737300 (030909_153743).
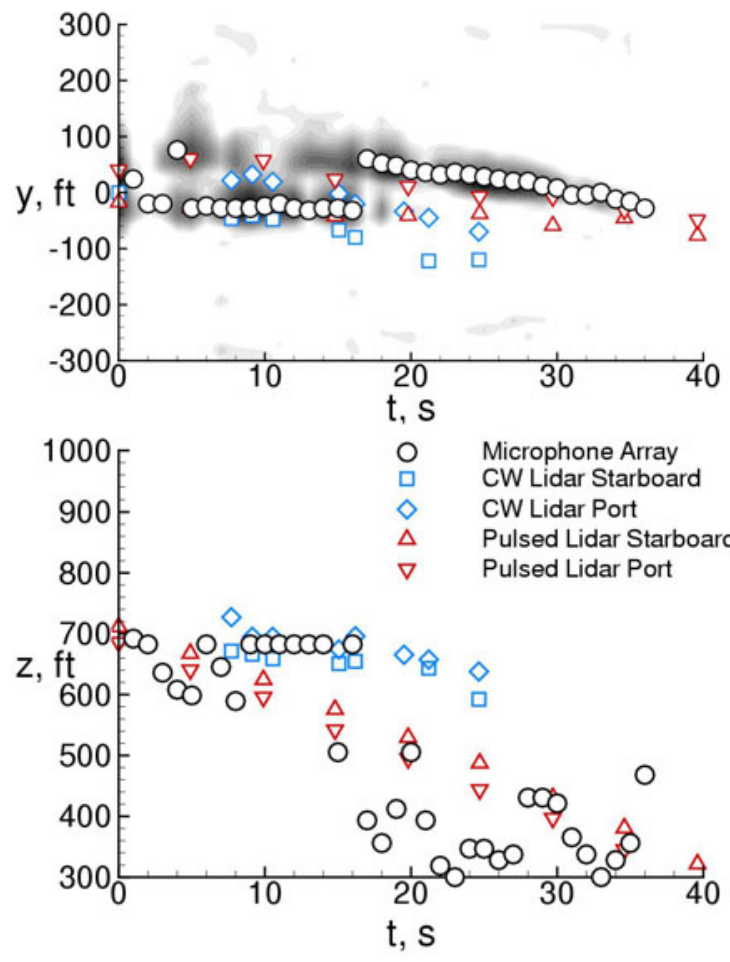

Figure 9. Tracking comparison for a Boeing 757200 (030909_101920). 

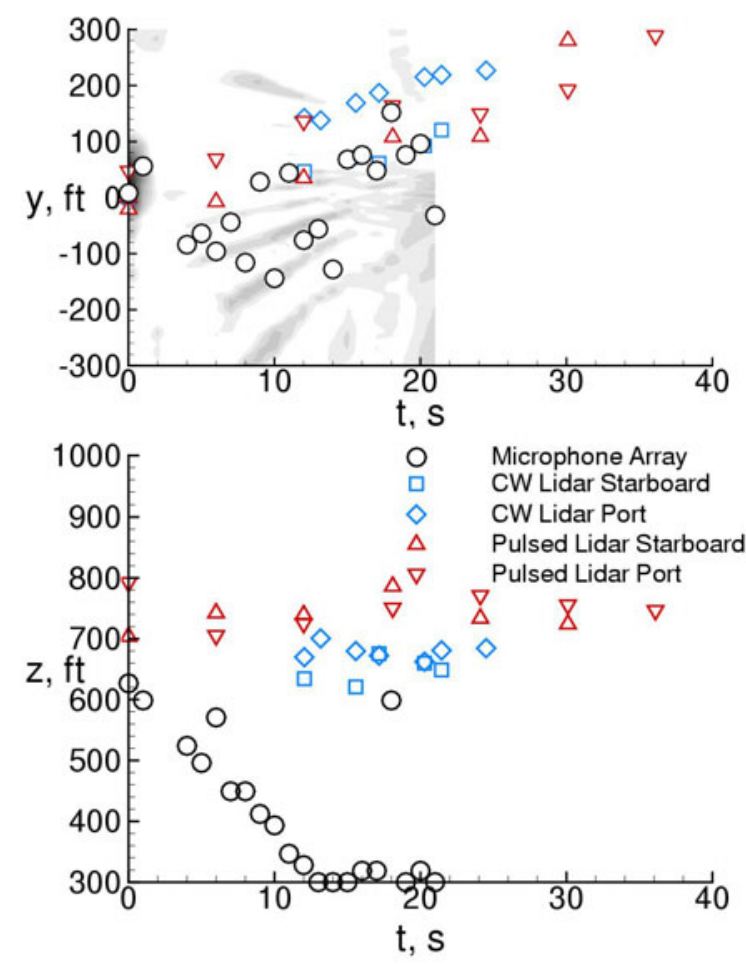

Figure 10. Tracking comparison for a Boeing 767300 (030916_105139).
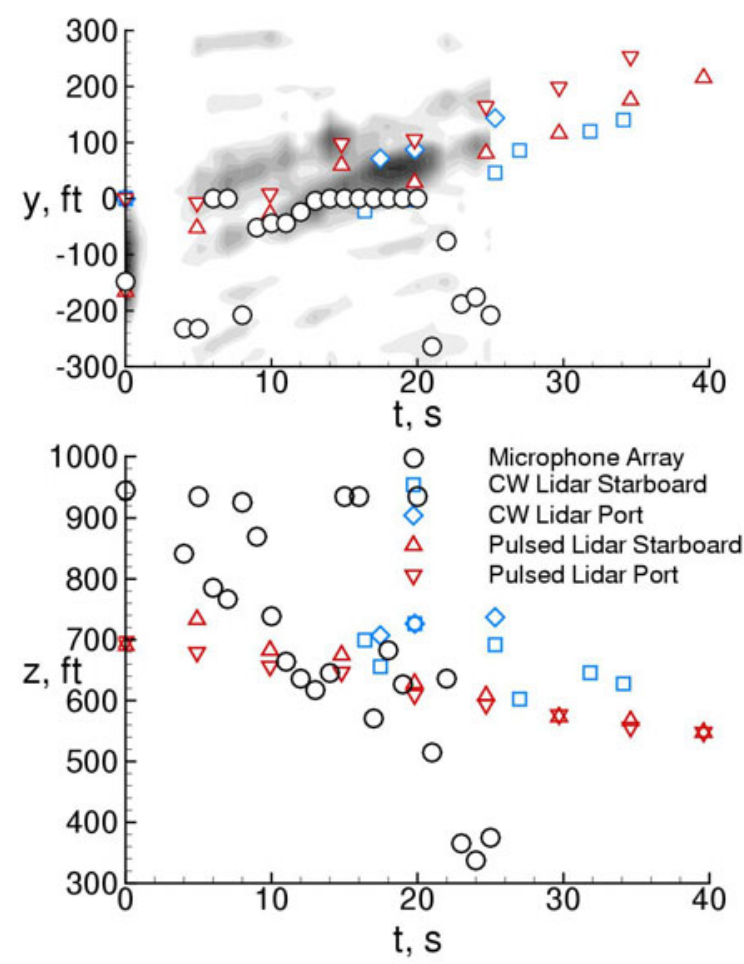

Figure 11. Tracking comparison for a McDonnell Douglas MD-81 (030919_084340).
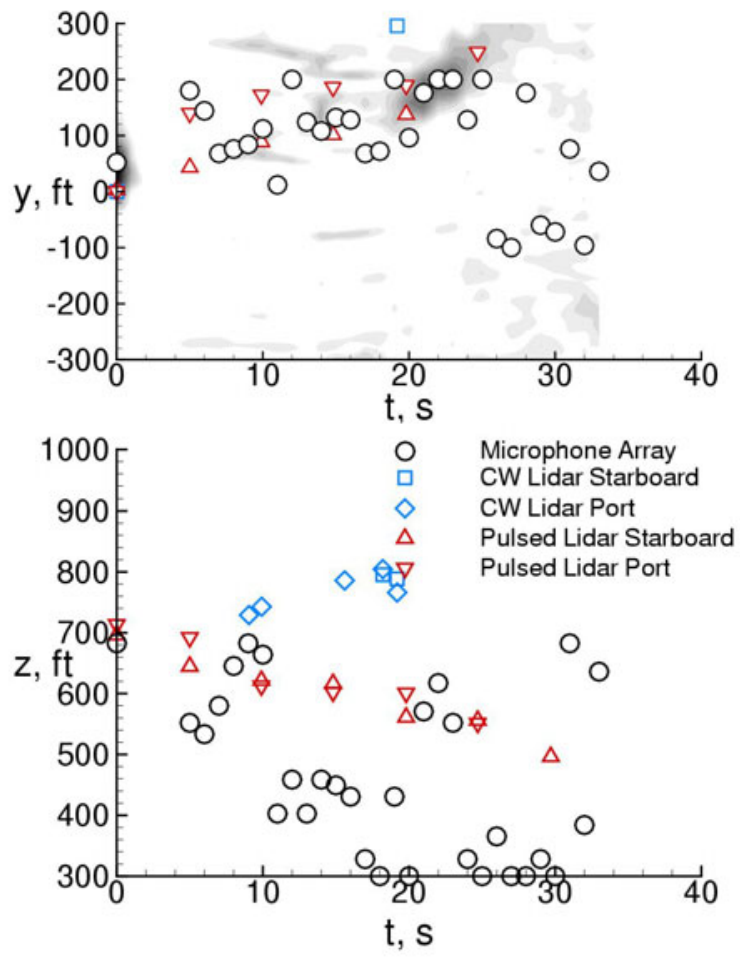

Figure 12. Tracking comparison for a McDonnell Douglas MD-82 (030909_164351).
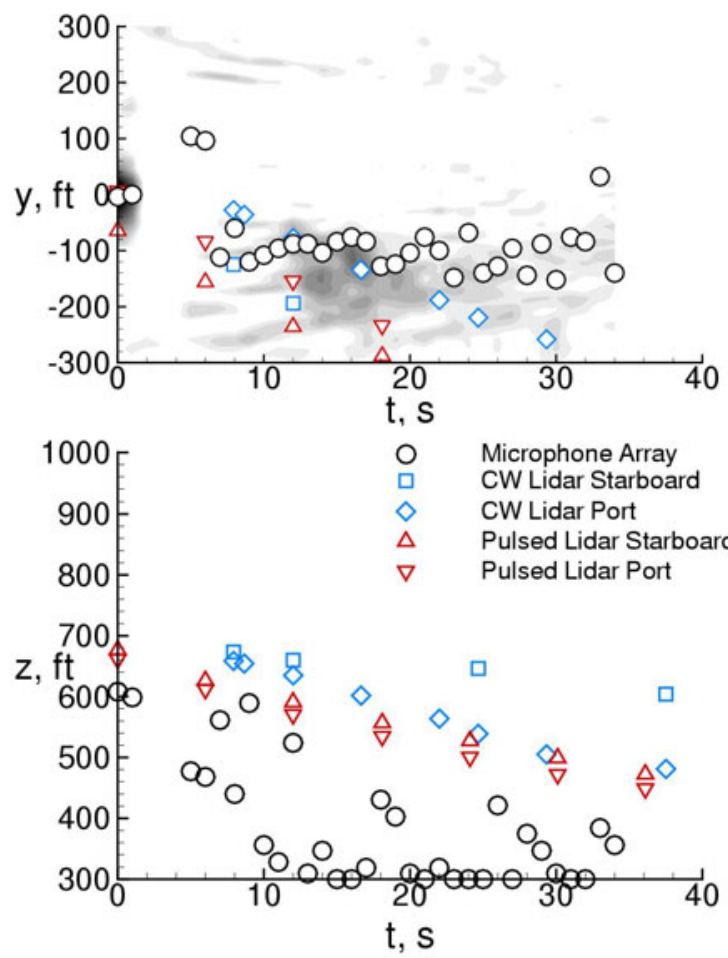

Figure 13. Tracking comparison for a McDonnell Douglas MD-87 (030919_121916). 


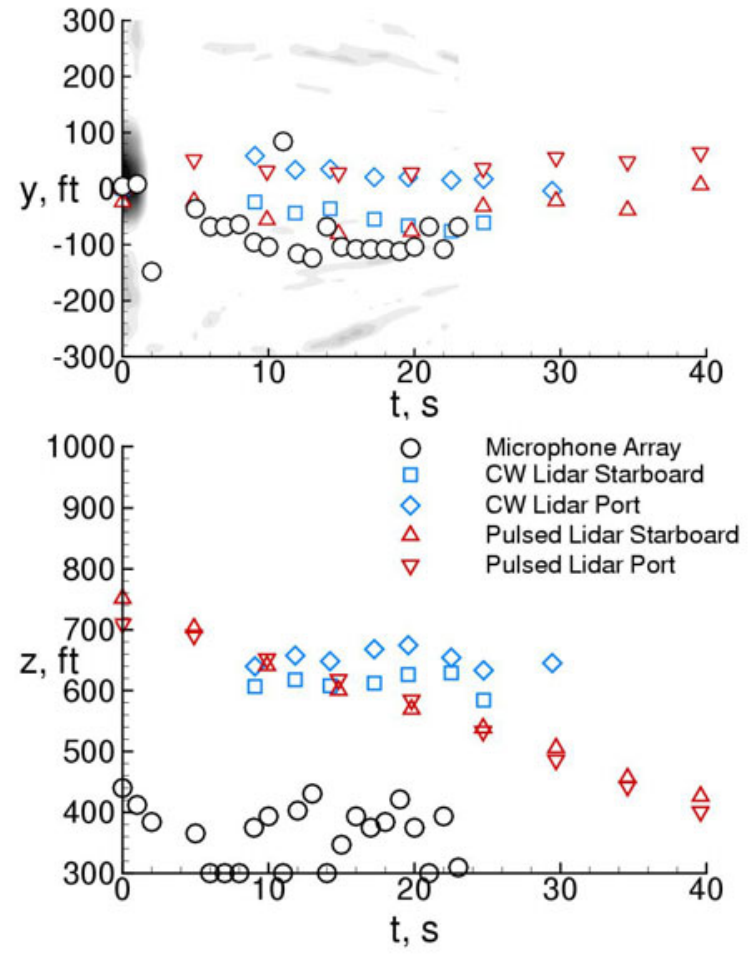

Figure 14. Tracking comparison for a Airbus A319 (030909_104210).
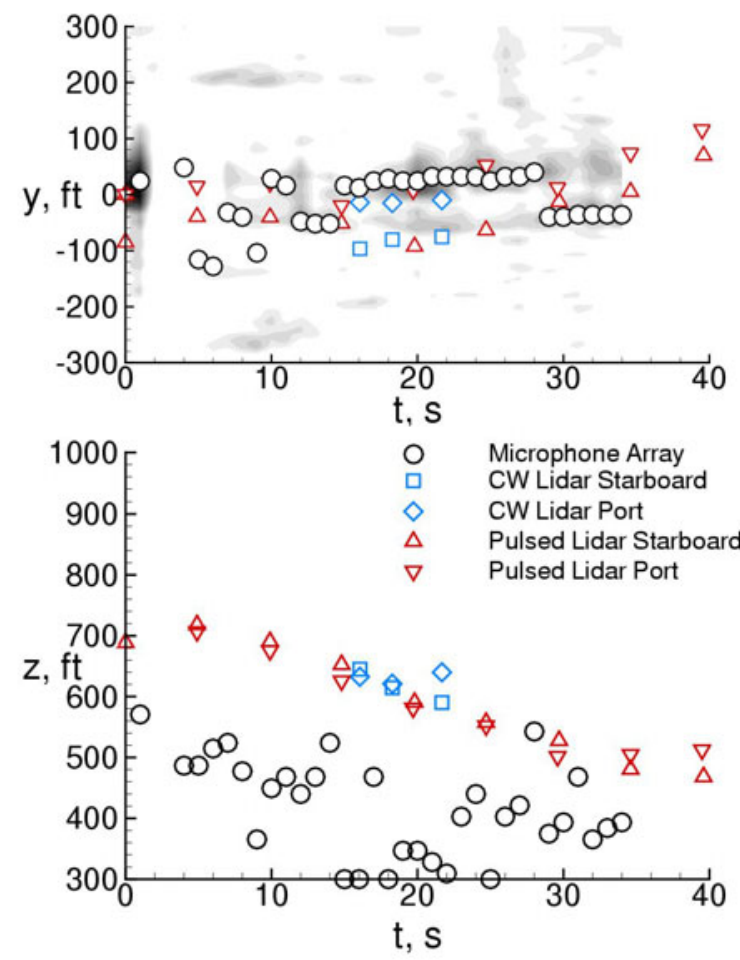

Figure 15. Tracking comparison for a Airbus A320 (030909_100626).
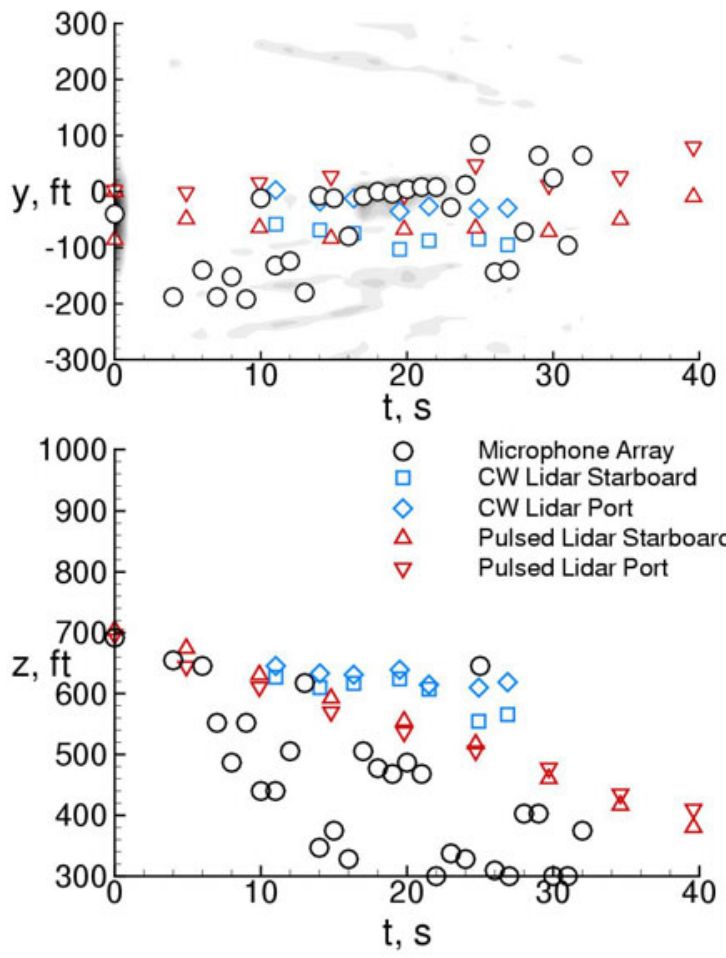

Figure 16. Tracking comparison for a Airbus A320 (030909_100750).
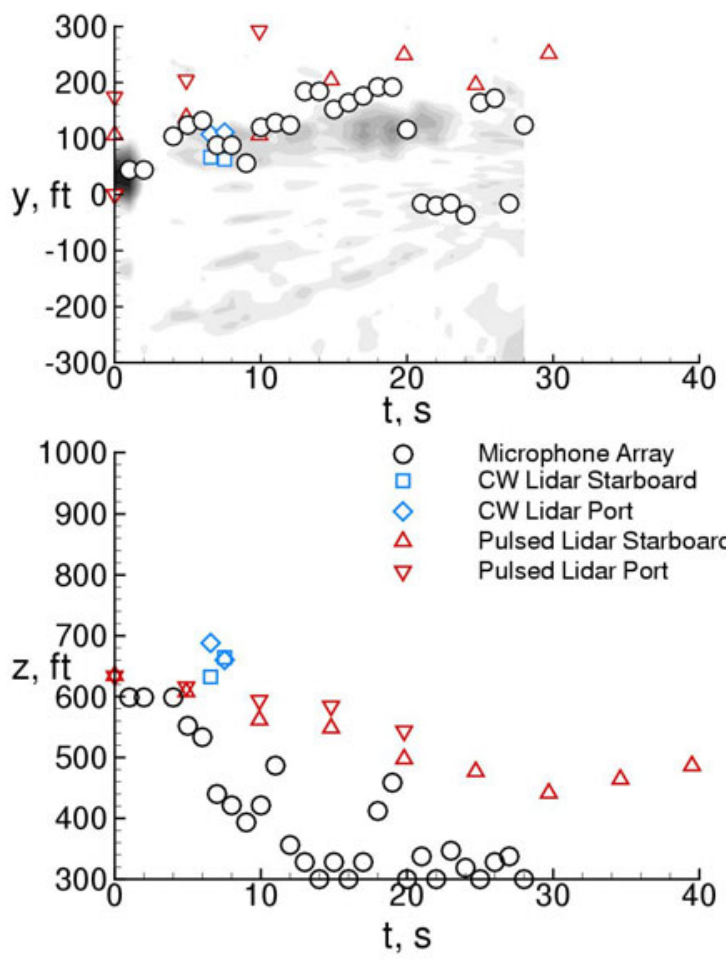

Figure 17. Tracking comparison for a Bombardier CRJ-200 (030919_084514). 


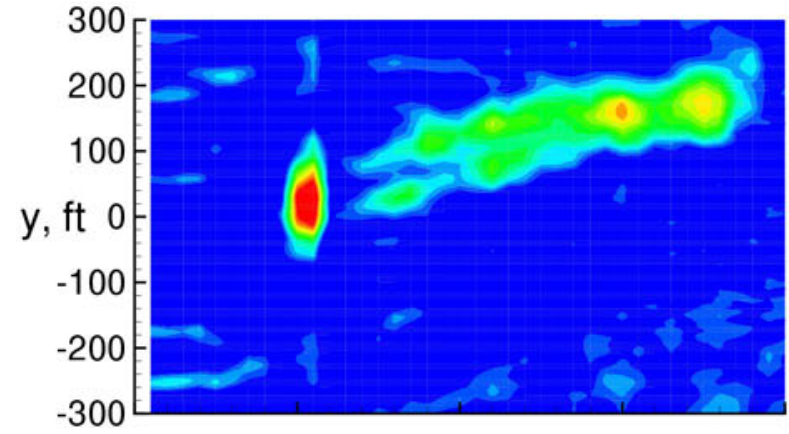

a) $100 \mathrm{~Hz}$ band

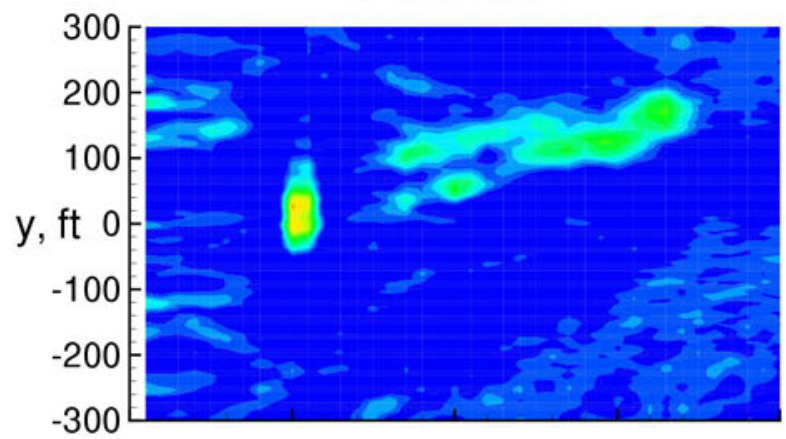

c) $160 \mathrm{~Hz}$ band

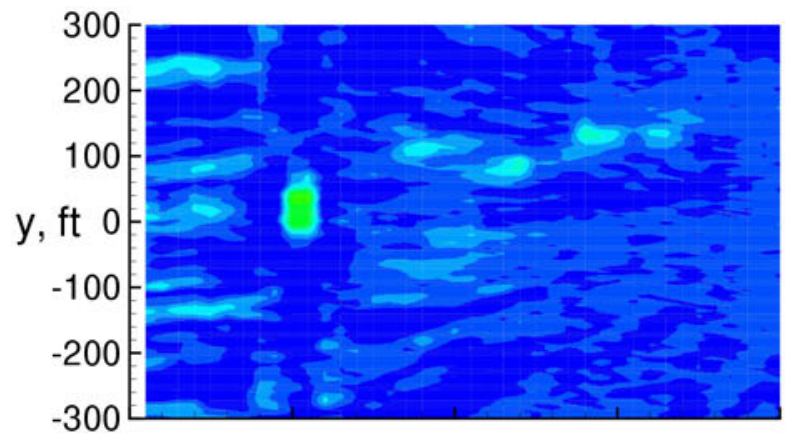

e) $250 \mathrm{~Hz}$ band

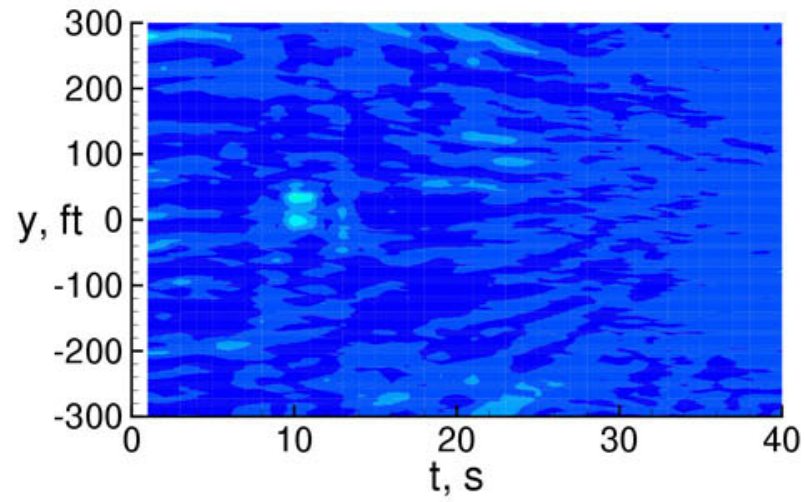

g) $400 \mathrm{~Hz}$ band

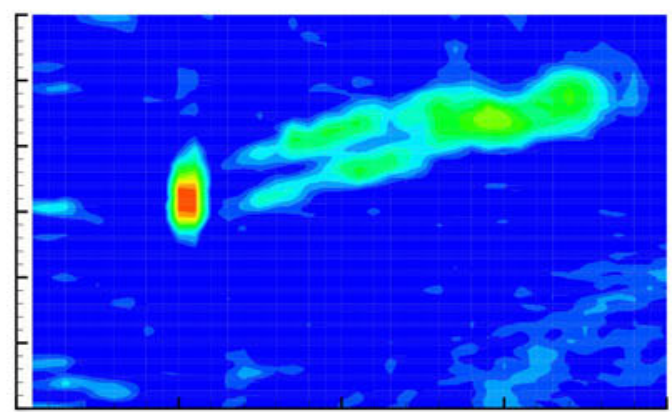

b) $125 \mathrm{~Hz}$ band

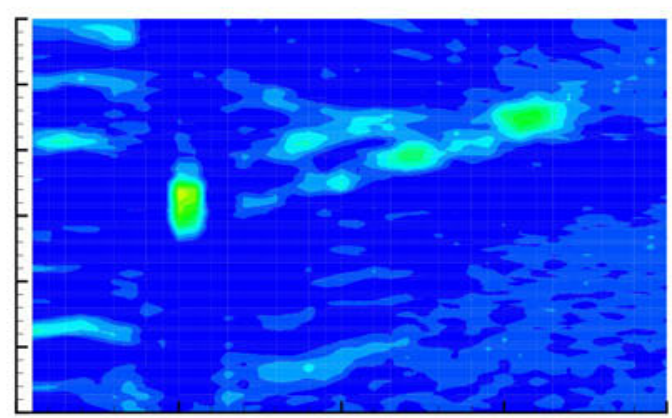

d) $200 \mathrm{~Hz}$ band

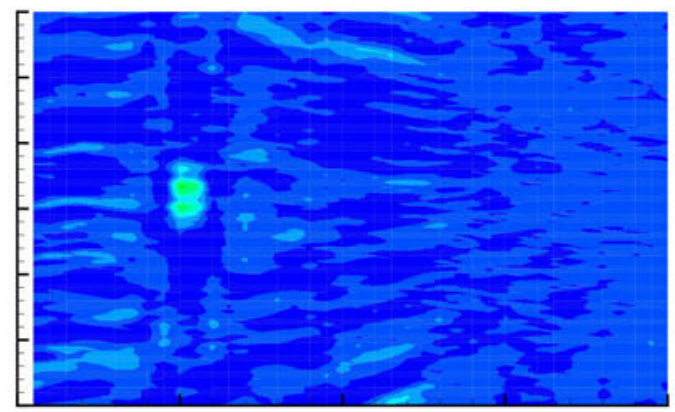

f) $315 \mathrm{~Hz}$ band

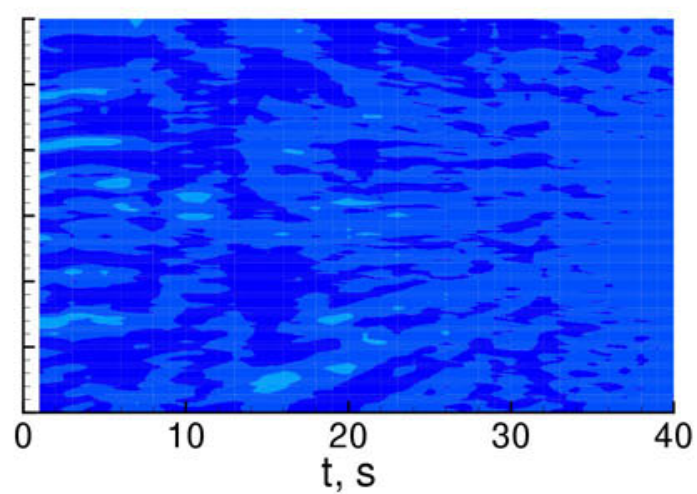

h) $500 \mathrm{~Hz}$ band

Figure 18. Spectral distribution of wake signal from a Boeing 737-300 (030915_130907). 


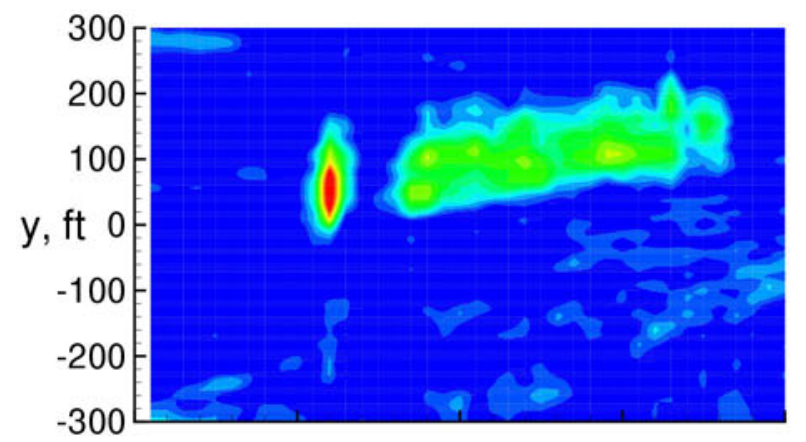

a) $100 \mathrm{~Hz}$ band

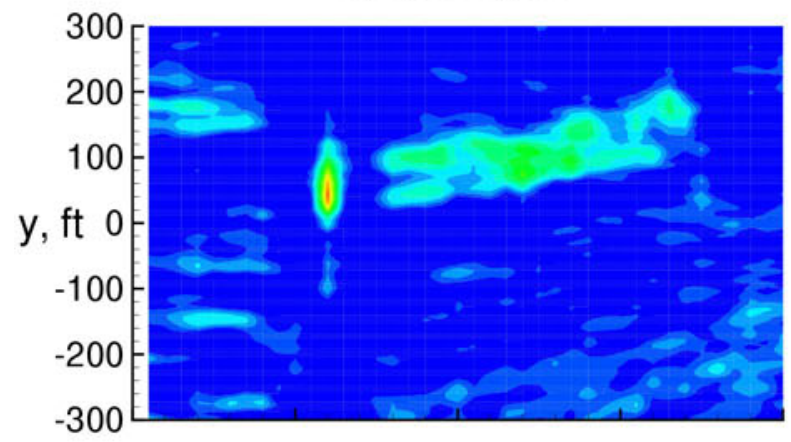

c) $160 \mathrm{~Hz}$ band

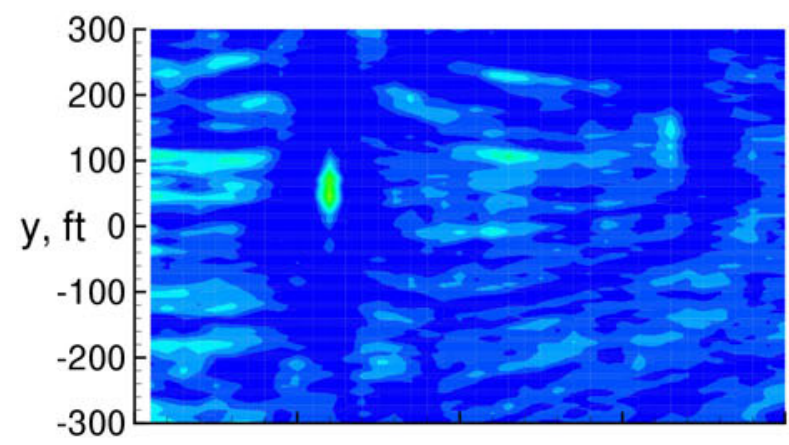

e) $250 \mathrm{~Hz}$ band

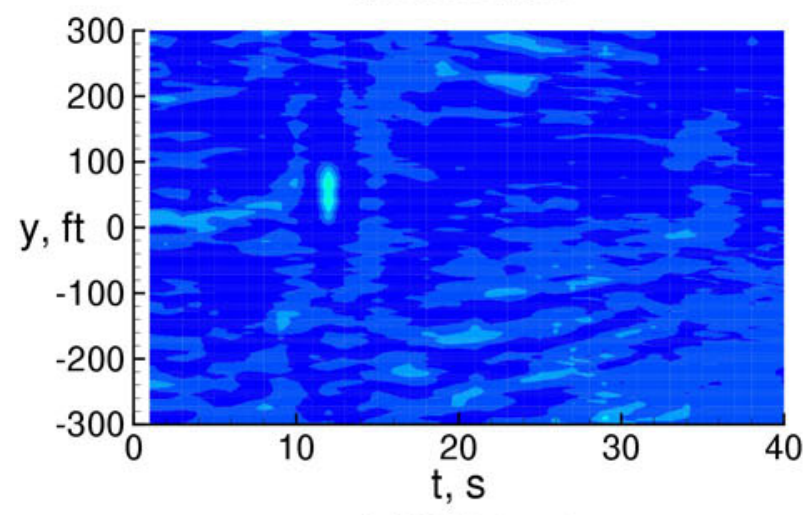

g) $400 \mathrm{~Hz}$ band

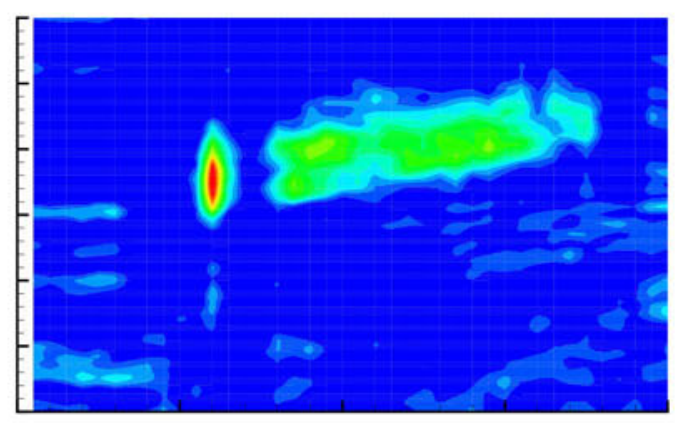

b) $125 \mathrm{~Hz}$ band

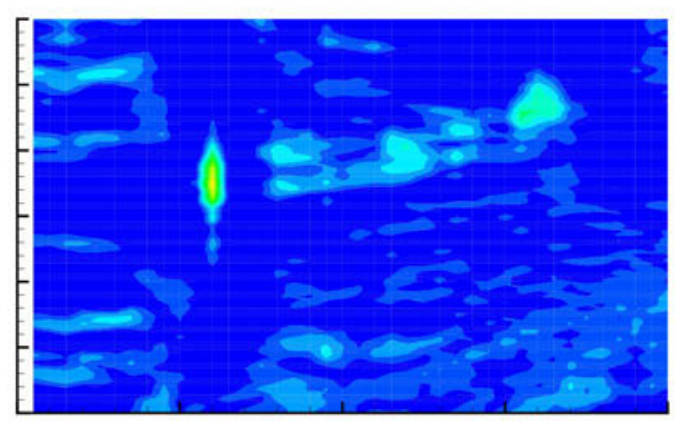

d) $200 \mathrm{~Hz}$ band

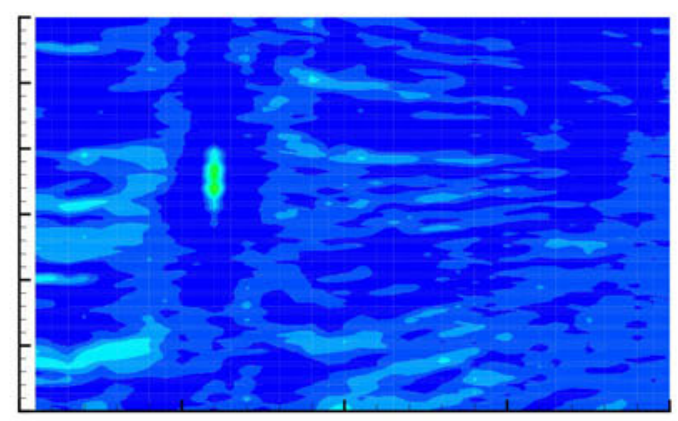

f) $315 \mathrm{~Hz}$ band

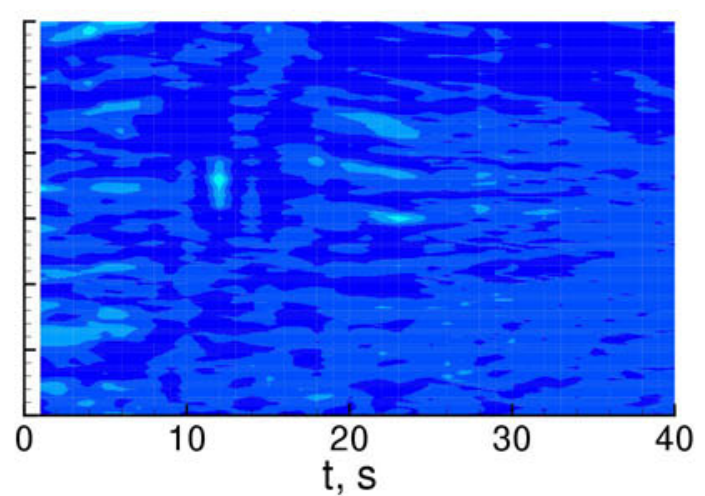

h) $500 \mathrm{~Hz}$ band

Figure 19. Spectral distribution of wake signal from a Boeing 737-300 (030916_104400). 


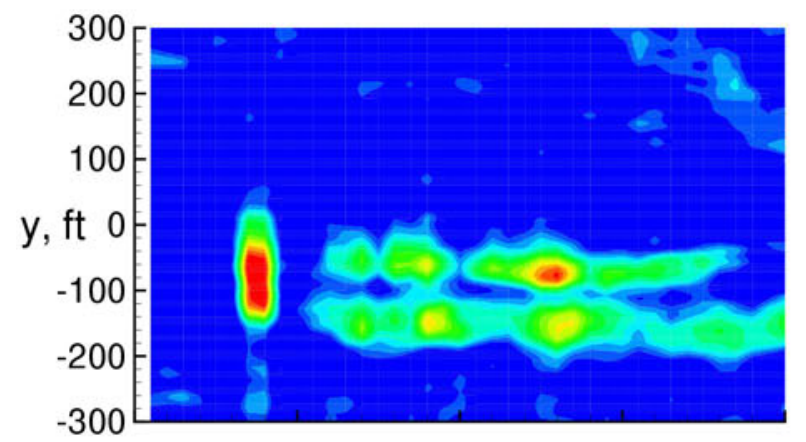

a) $100 \mathrm{~Hz}$ band

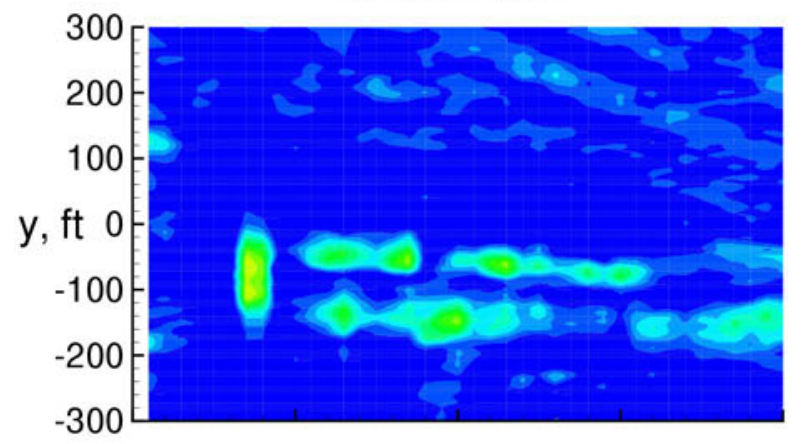

c) $160 \mathrm{~Hz}$ band

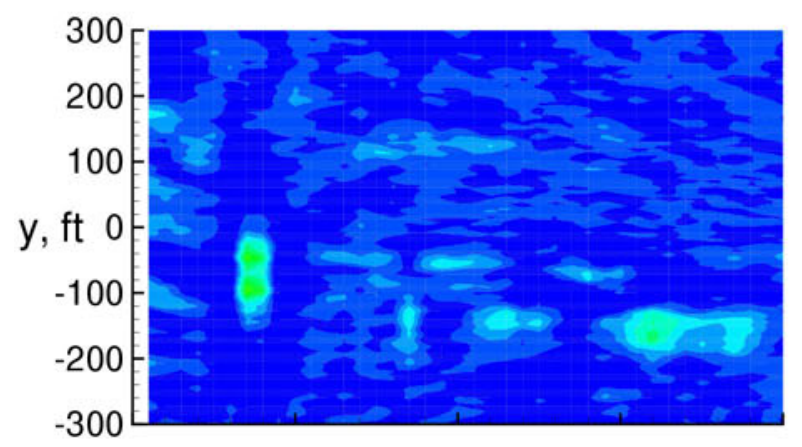

e) $250 \mathrm{~Hz}$ band

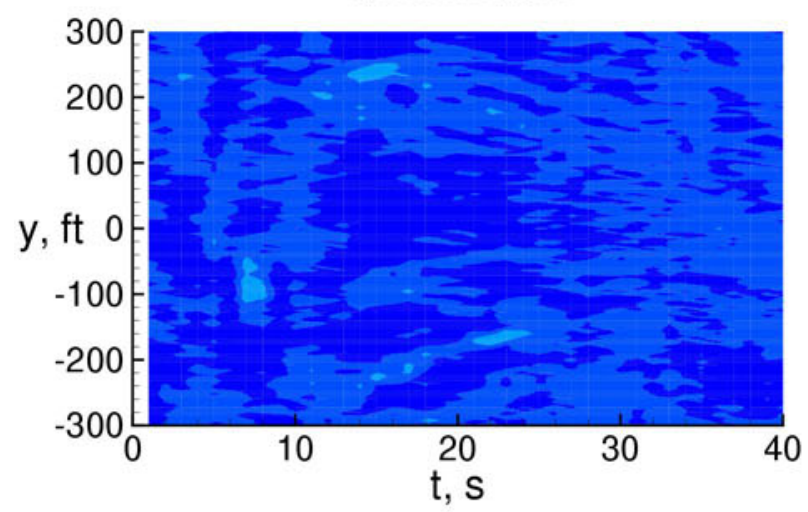

g) $400 \mathrm{~Hz}$ band

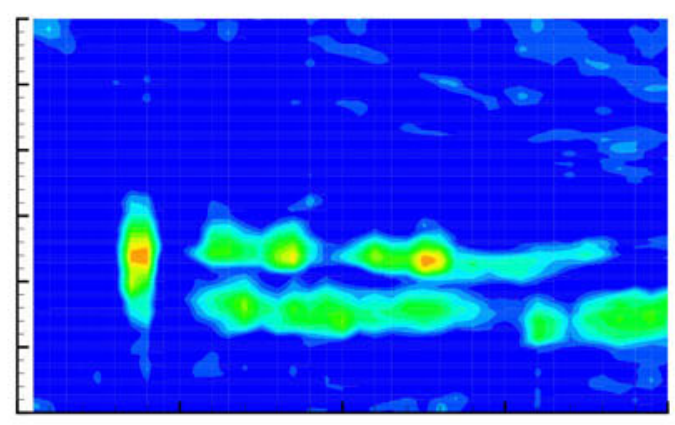

b) $125 \mathrm{~Hz}$ band

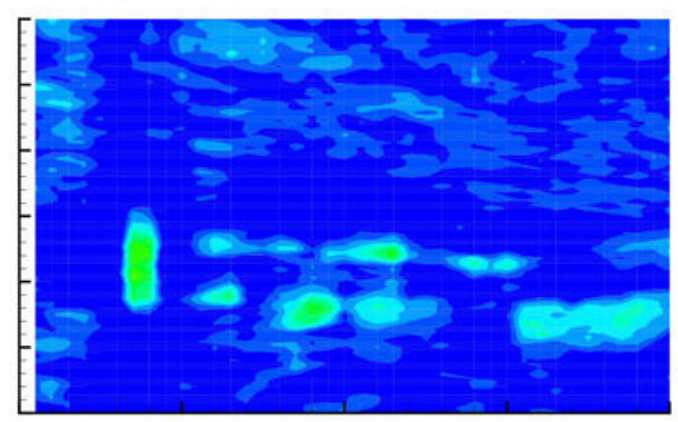

d) $200 \mathrm{~Hz}$ band

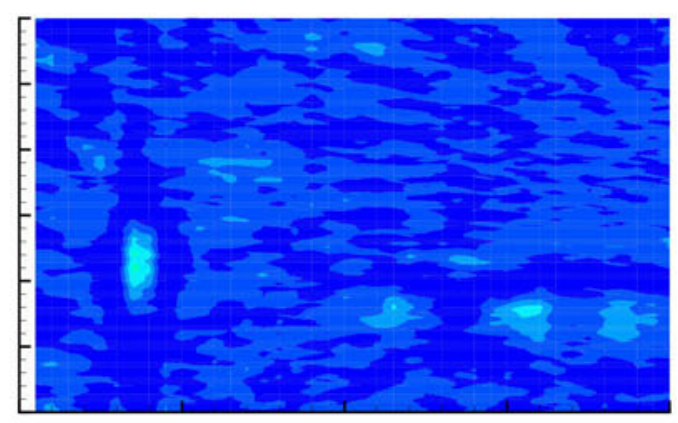

f) $315 \mathrm{~Hz}$ band

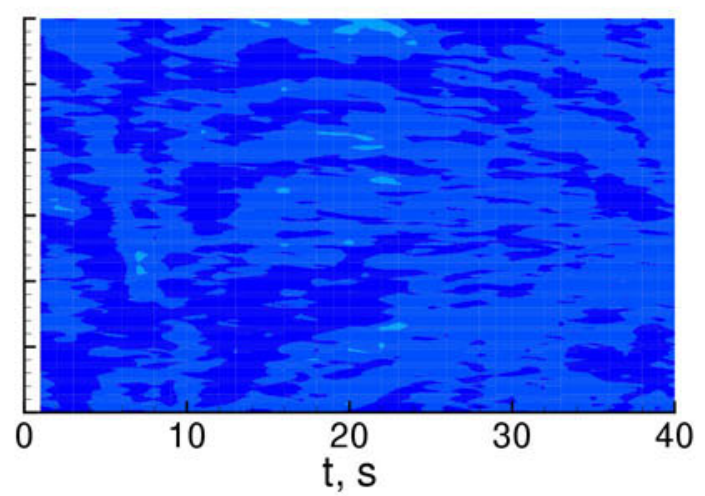

h) $500 \mathrm{~Hz}$ band

Figure 20. Spectral distribution of wake signal from a Boeing 757-200 (030909_153743). 


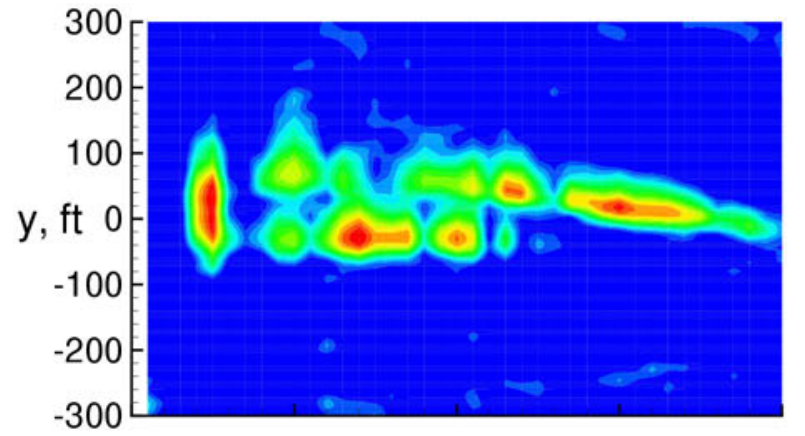

a) $100 \mathrm{~Hz}$ band

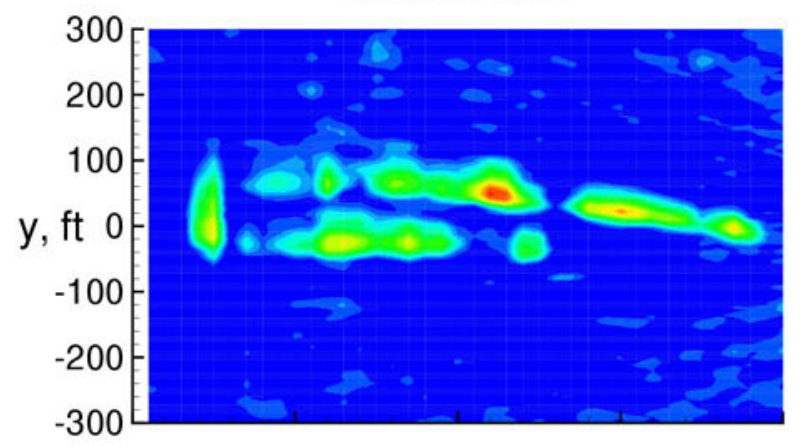

c) $160 \mathrm{~Hz}$ band

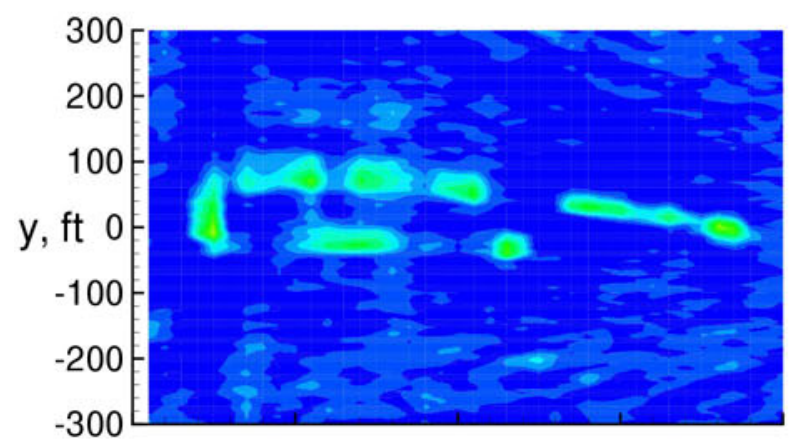

e) $250 \mathrm{~Hz}$ band

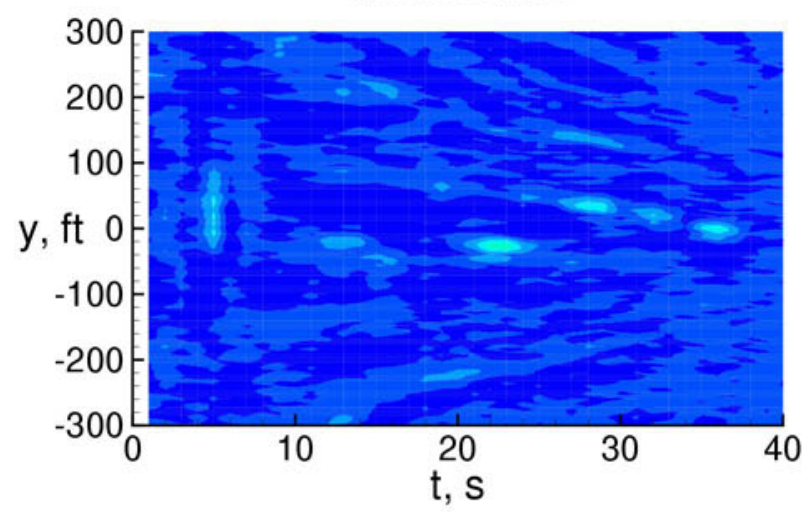

g) $400 \mathrm{~Hz}$ band

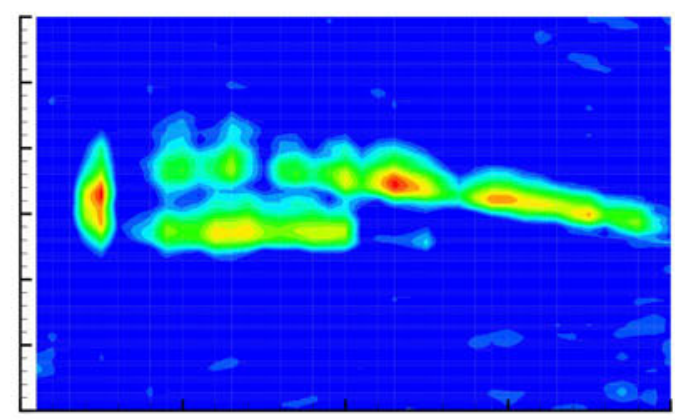

b) $125 \mathrm{~Hz}$ band

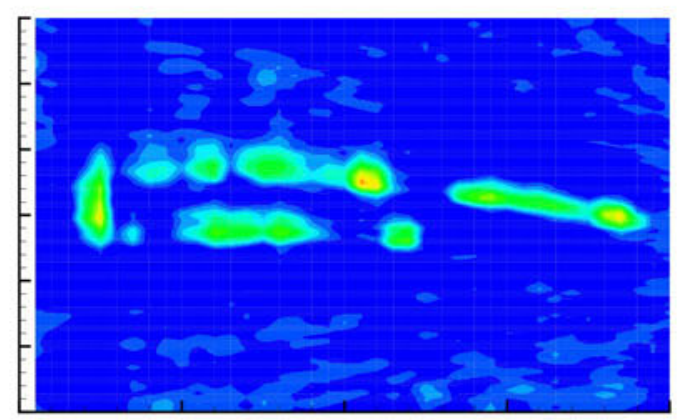

d) $200 \mathrm{~Hz}$ band

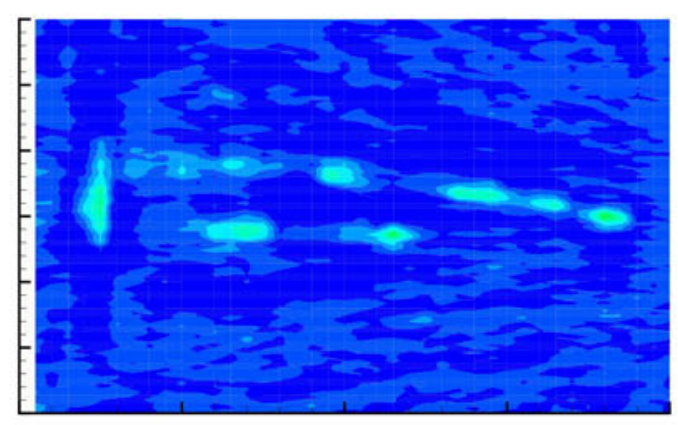

f) $315 \mathrm{~Hz}$ band

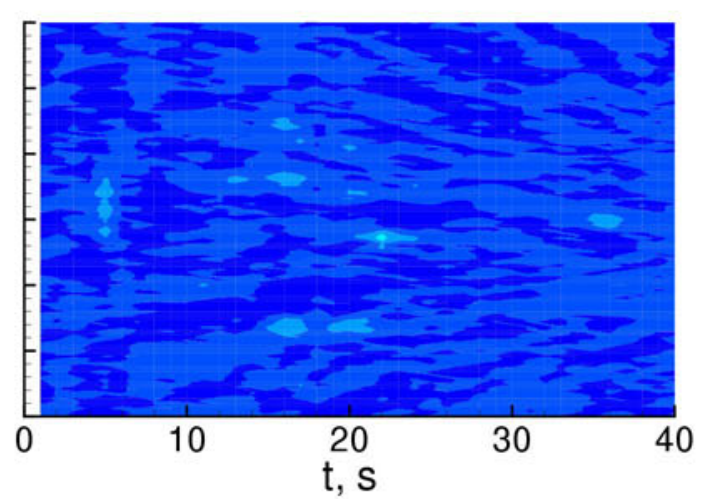

h) $500 \mathrm{~Hz}$ band

Figure 21. Spectral distribution of wake signal from a Boeing 757-200 (030909_101920). 


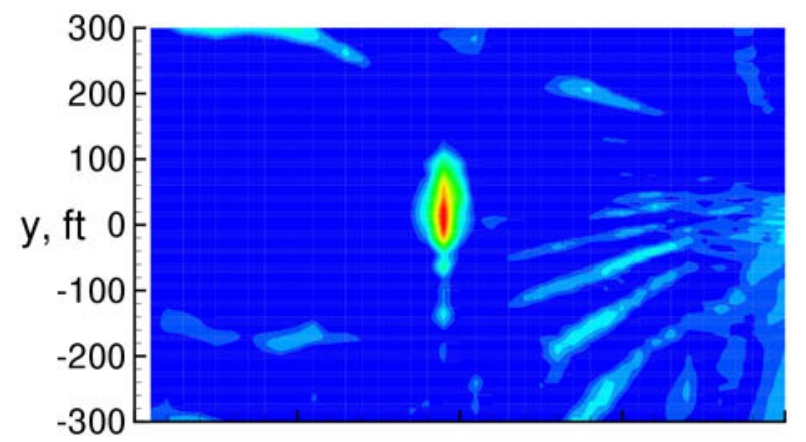

a) $100 \mathrm{~Hz}$ band

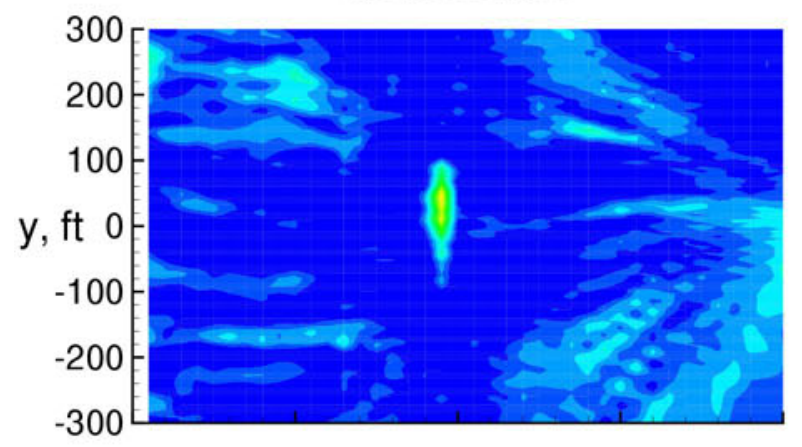

c) $160 \mathrm{~Hz}$ band

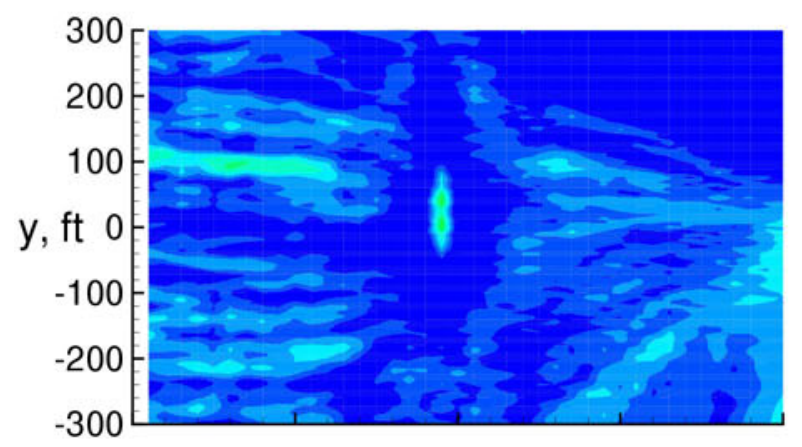

e) $250 \mathrm{~Hz}$ band

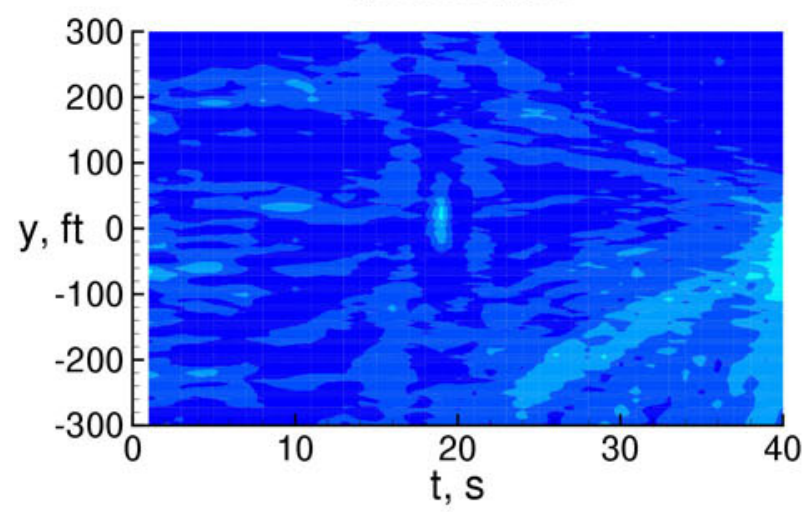

g) $400 \mathrm{~Hz}$ band

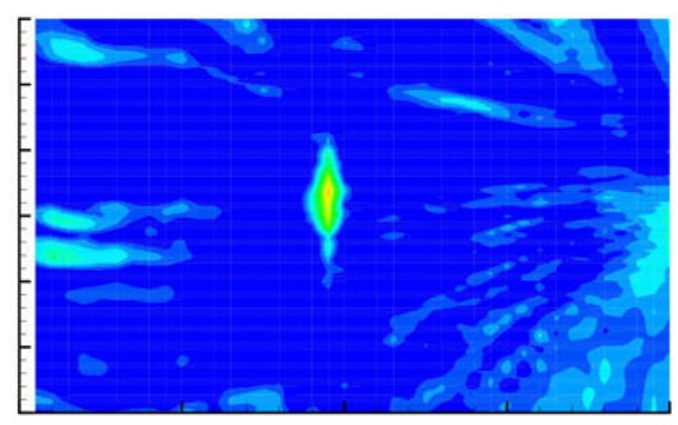

b) $125 \mathrm{~Hz}$ band

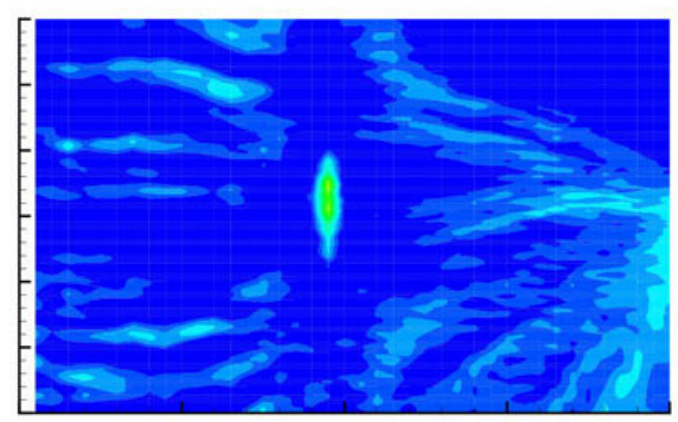

d) $200 \mathrm{~Hz}$ band

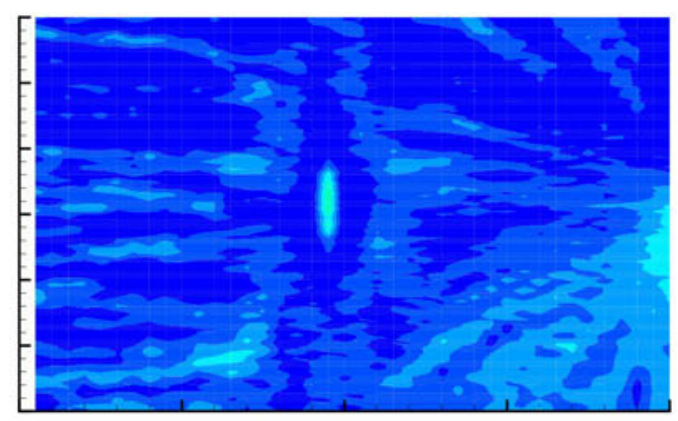

f) $315 \mathrm{~Hz}$ band

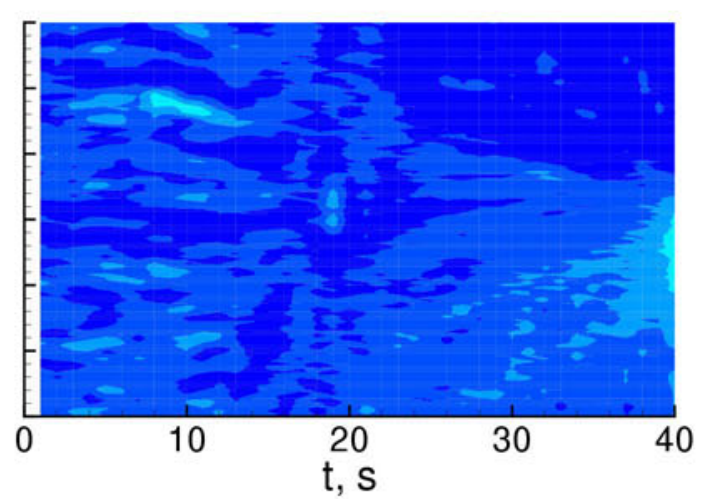

h) $500 \mathrm{~Hz}$ band

Figure 22. Spectral distribution of wake signal from a Boeing 767-300 (030916_105139). 


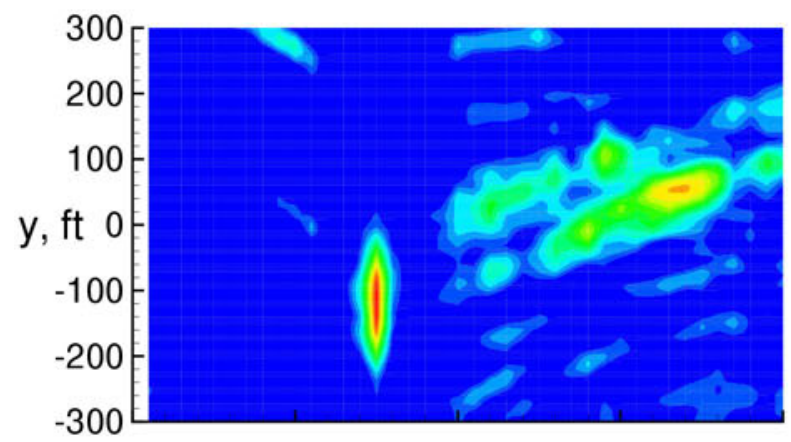

a) $100 \mathrm{~Hz}$ band

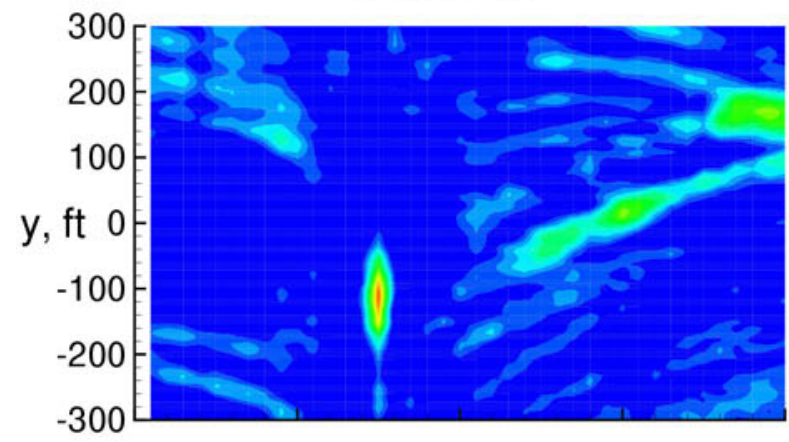

c) $160 \mathrm{~Hz}$ band

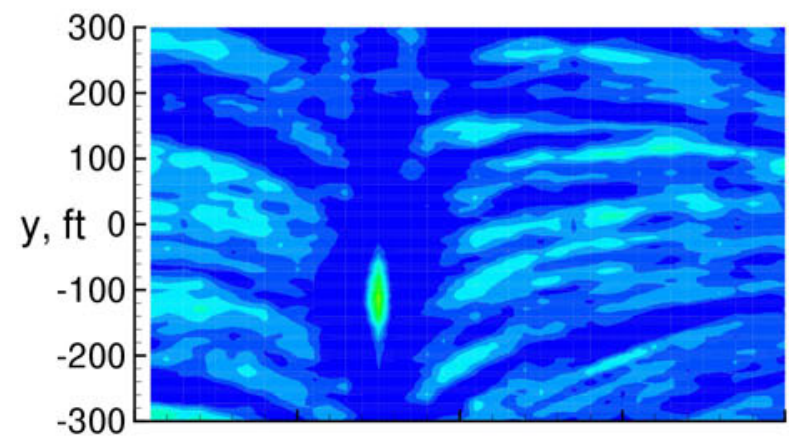

e) $250 \mathrm{~Hz}$ band

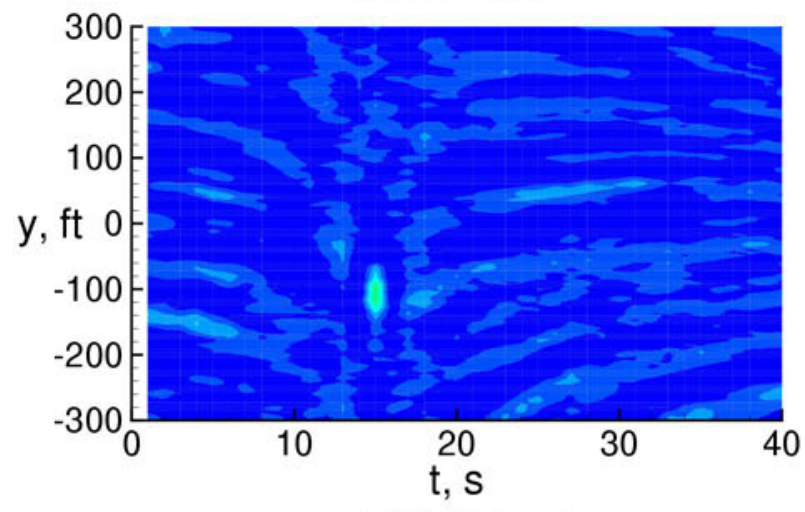

g) $400 \mathrm{~Hz}$ band

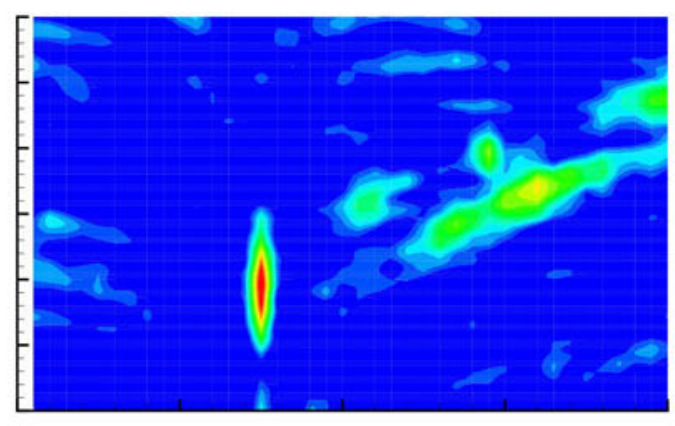

b) $125 \mathrm{~Hz}$ band

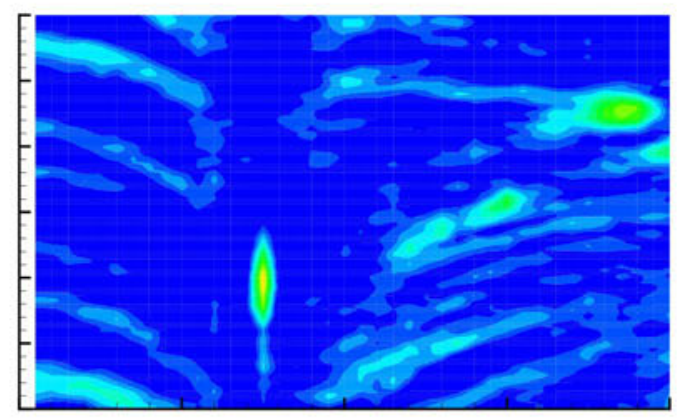

d) $200 \mathrm{~Hz}$ band

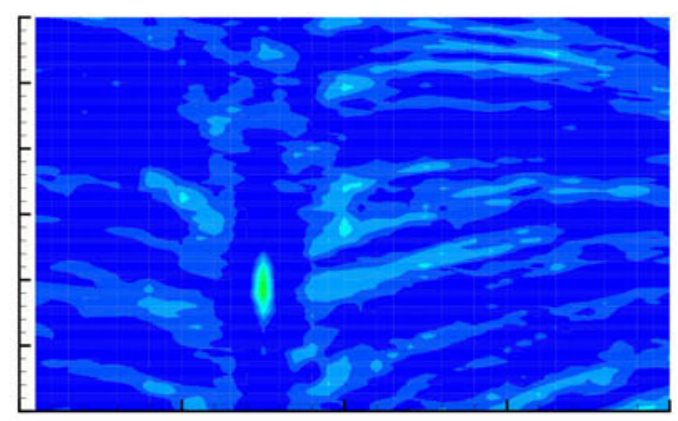

f) $315 \mathrm{~Hz}$ band

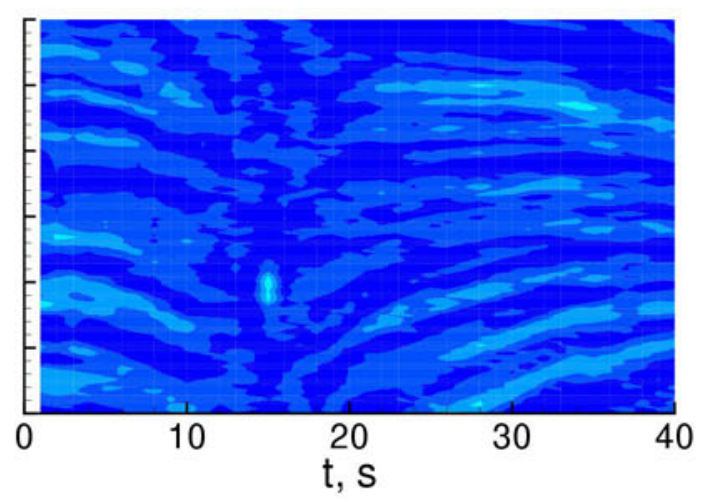

h) $500 \mathrm{~Hz}$ band

Figure 23. Spectral distribution of wake signal from a McDonnell Douglas MD-81 (030919_084340). 


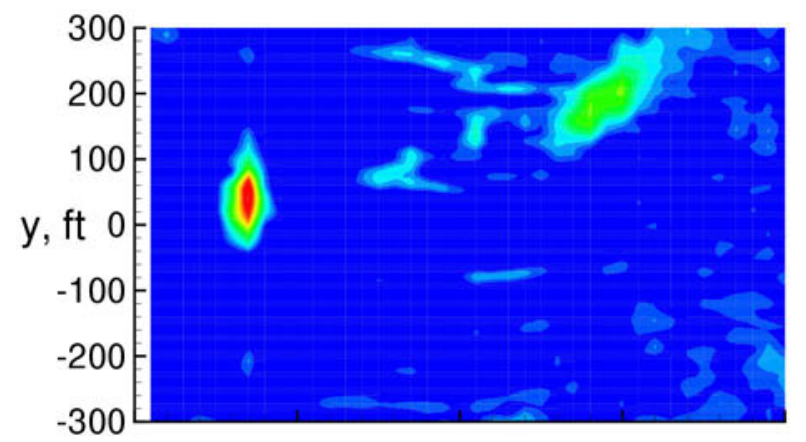

a) $100 \mathrm{~Hz}$ band

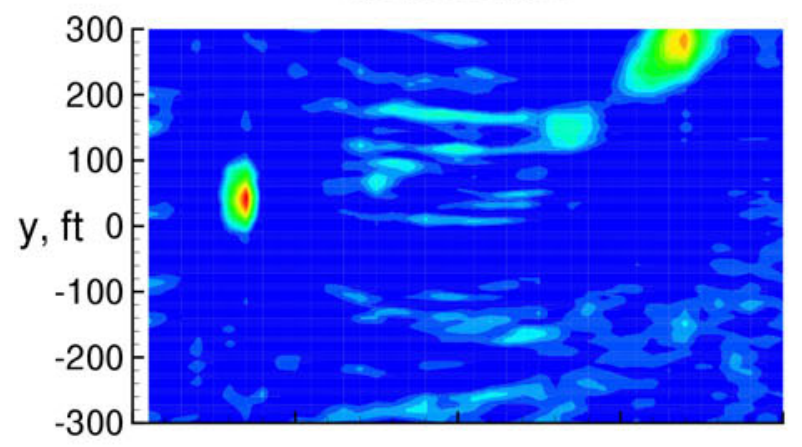

c) $160 \mathrm{~Hz}$ band

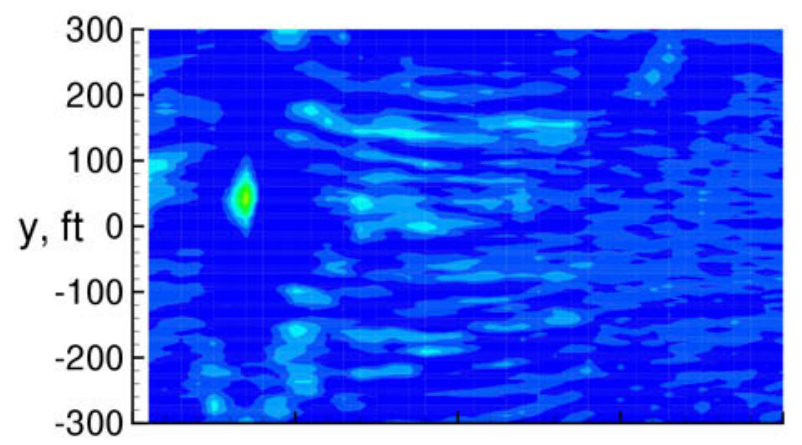

e) $250 \mathrm{~Hz}$ band

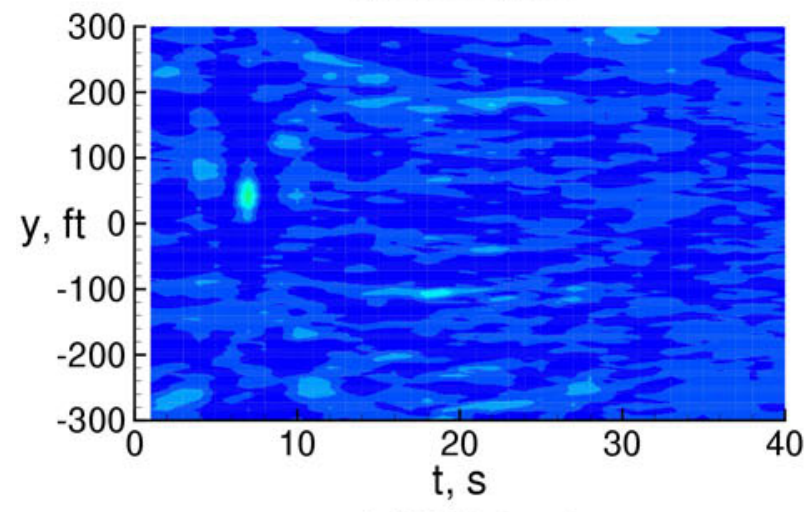

g) $400 \mathrm{~Hz}$ band

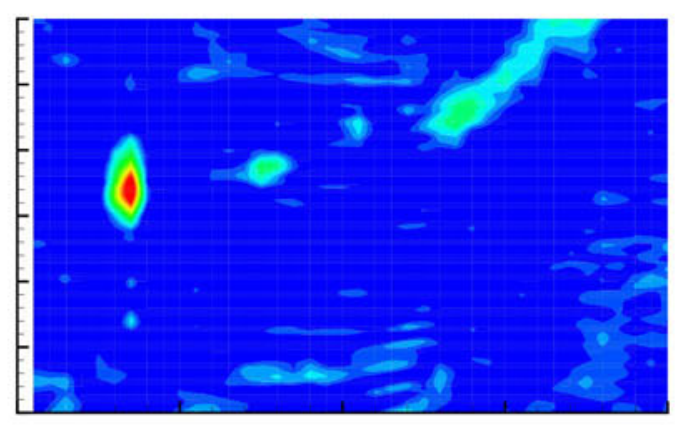

b) $125 \mathrm{~Hz}$ band

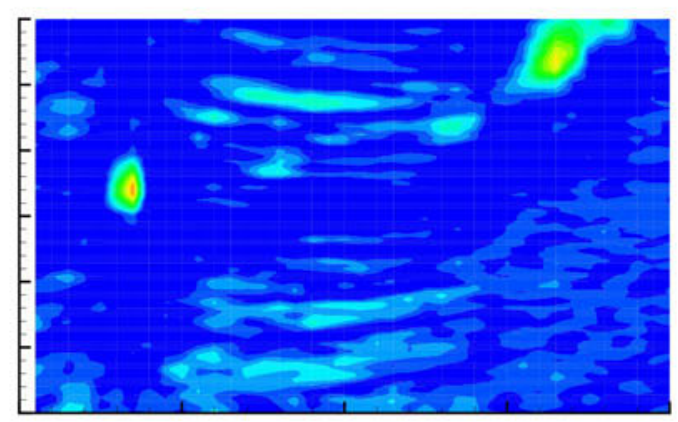

d) $200 \mathrm{~Hz}$ band

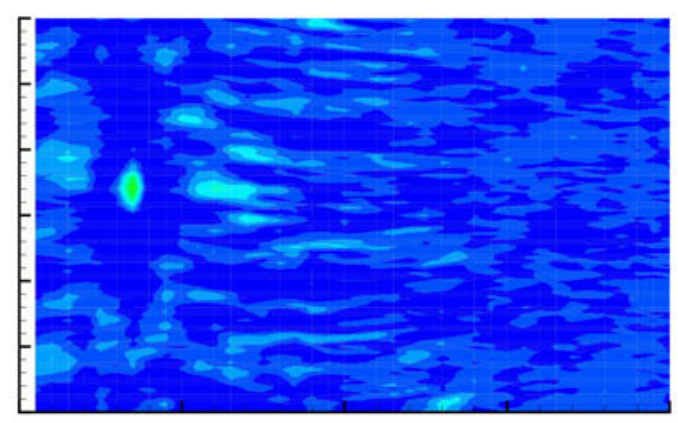

f) $315 \mathrm{~Hz}$ band

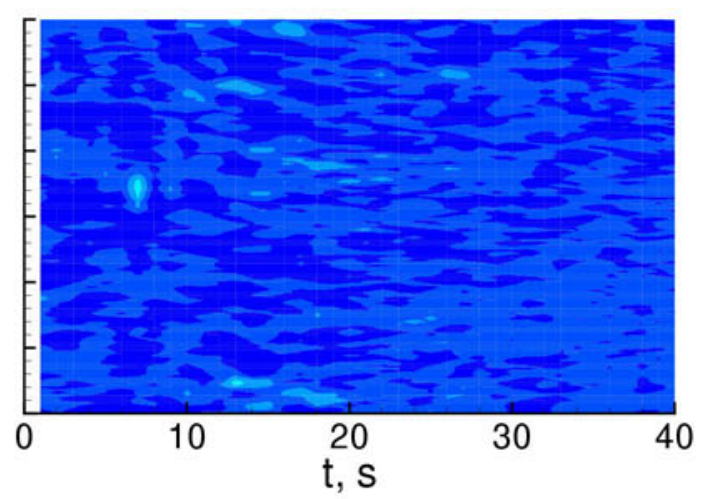

h) $500 \mathrm{~Hz}$ band

Figure 24. Spectral distribution of wake signal from a McDonnell Douglas MD-82 (030909_164351). 


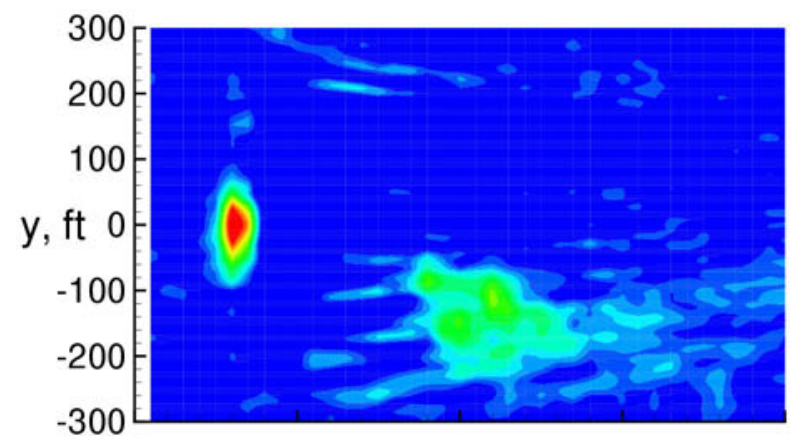

a) $100 \mathrm{~Hz}$ band

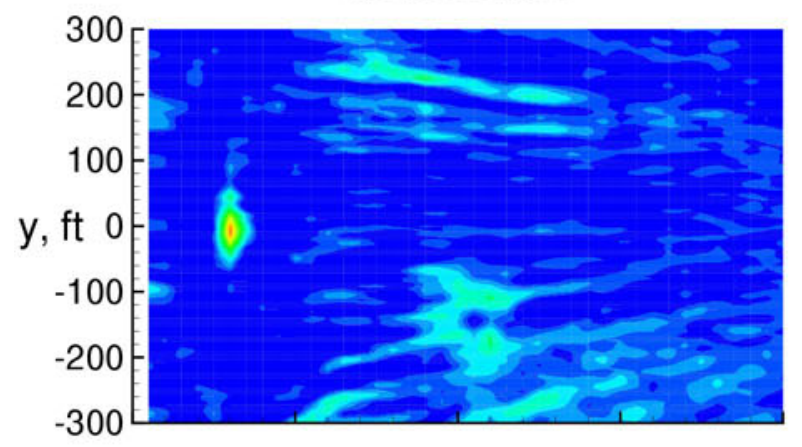

c) $160 \mathrm{~Hz}$ band

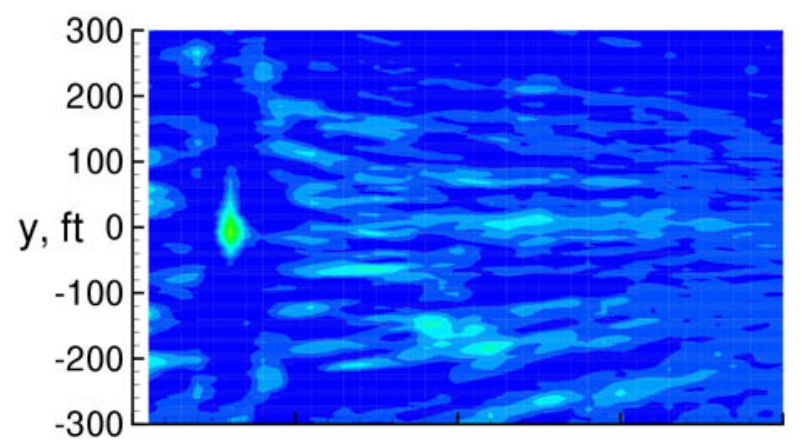

e) $250 \mathrm{~Hz}$ band

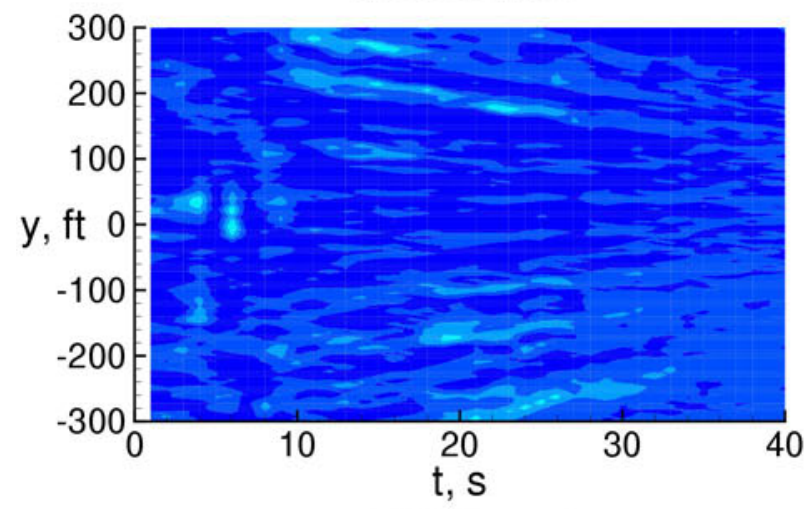

g) $400 \mathrm{~Hz}$ band

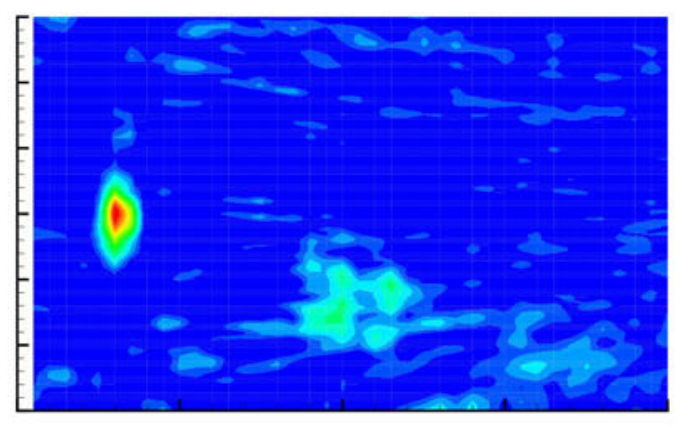

b) $125 \mathrm{~Hz}$ band

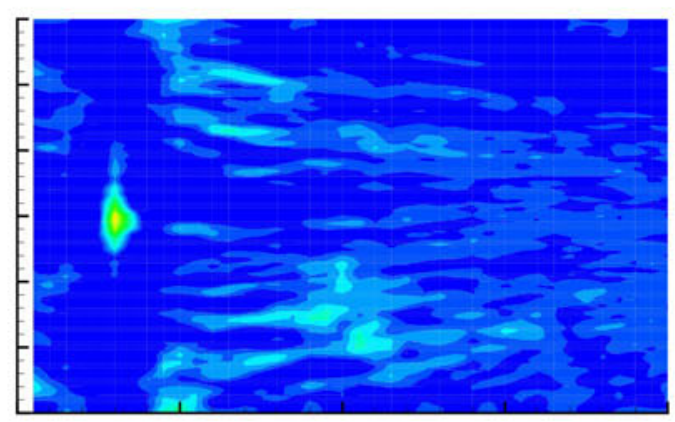

d) $200 \mathrm{~Hz}$ band

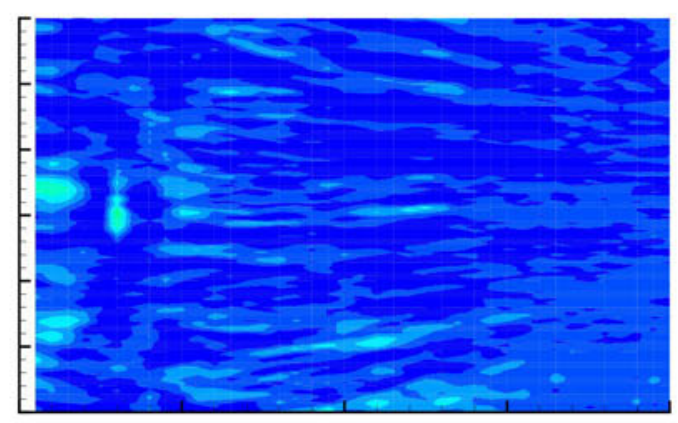

f) $315 \mathrm{~Hz}$ band

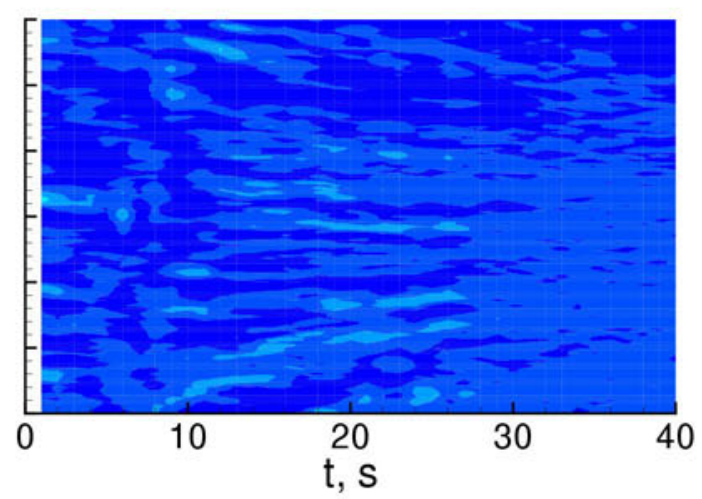

h) $500 \mathrm{~Hz}$ band

Figure 25. Spectral distribution of wake signal from a McDonnell Douglas MD-87 (030919_121916). 


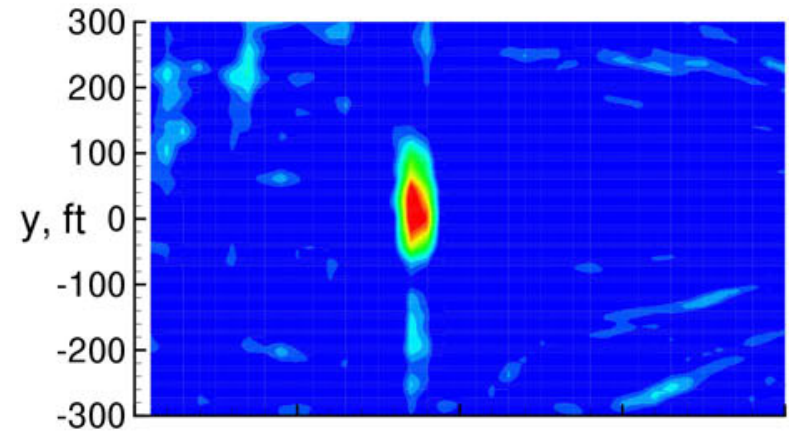

a) $100 \mathrm{~Hz}$ band

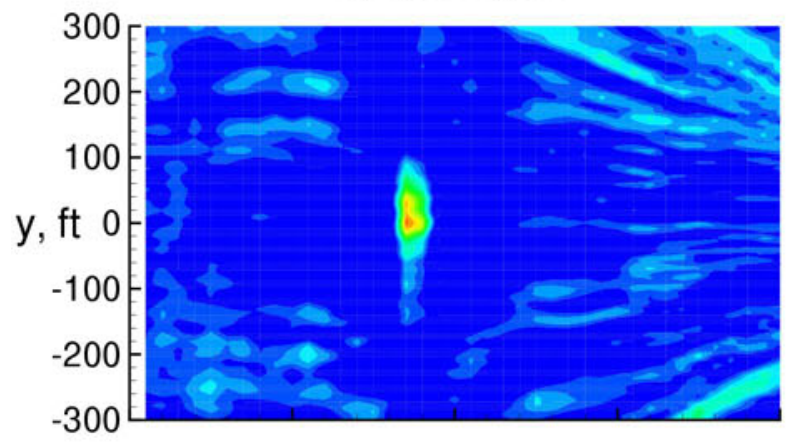

c) $160 \mathrm{~Hz}$ band

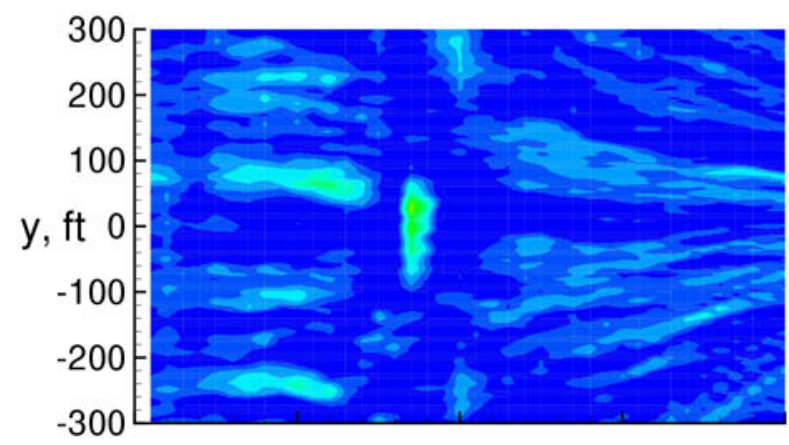

e) $250 \mathrm{~Hz}$ band

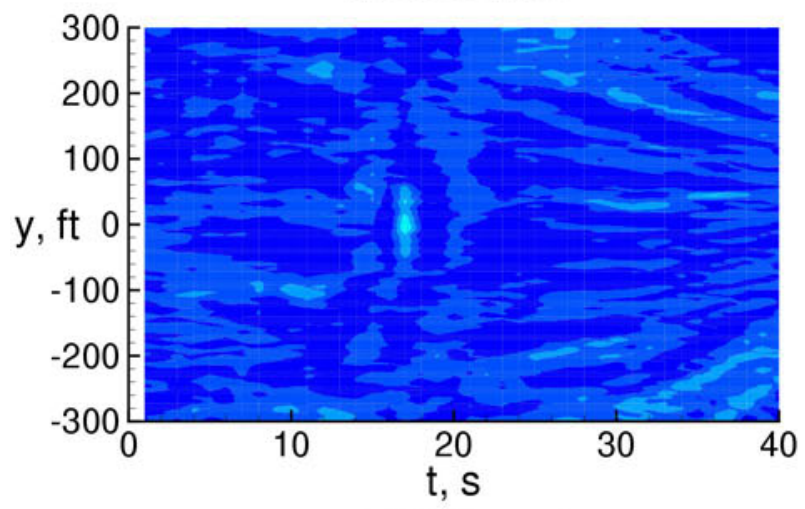

g) $400 \mathrm{~Hz}$ band

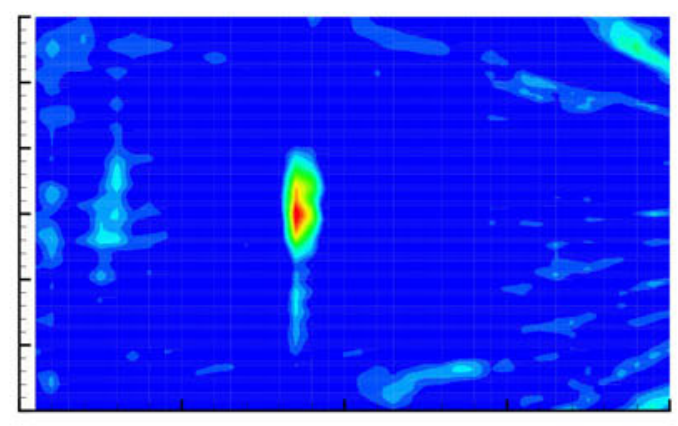

b) $125 \mathrm{~Hz}$ band

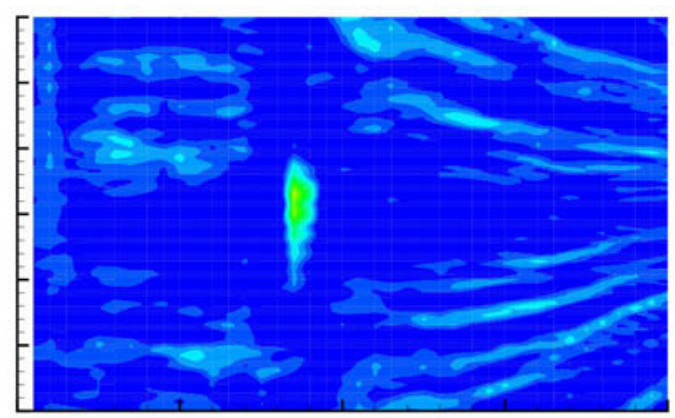

d) $200 \mathrm{~Hz}$ band

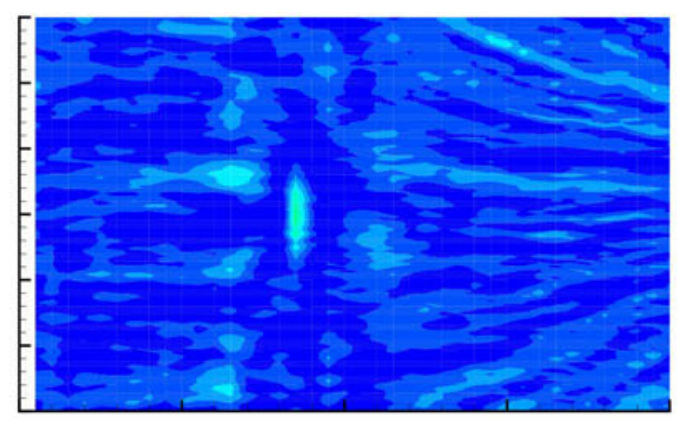

f) $315 \mathrm{~Hz}$ band

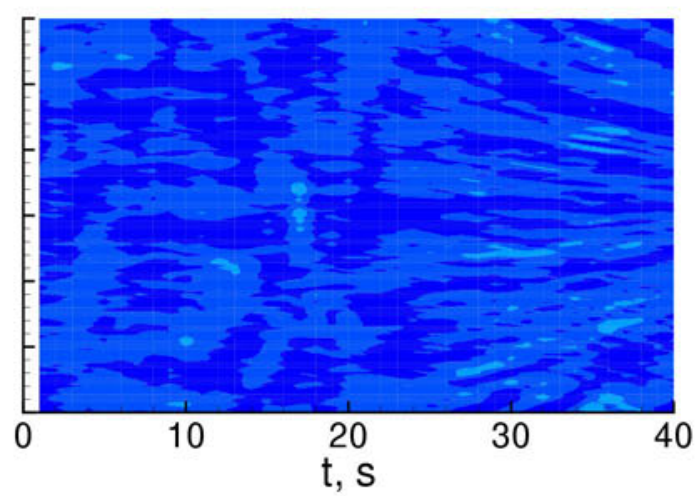

h) $500 \mathrm{~Hz}$ band

Figure 26. Spectral distribution of wake signal from an Airbus A319 (030909_104210). 


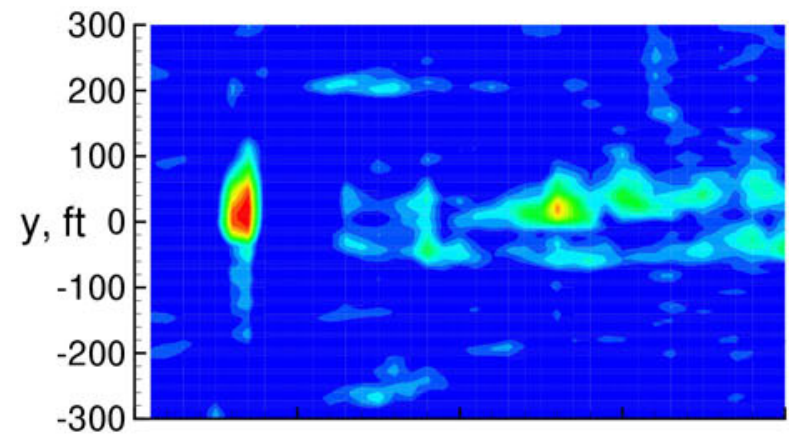

a) $100 \mathrm{~Hz}$ band

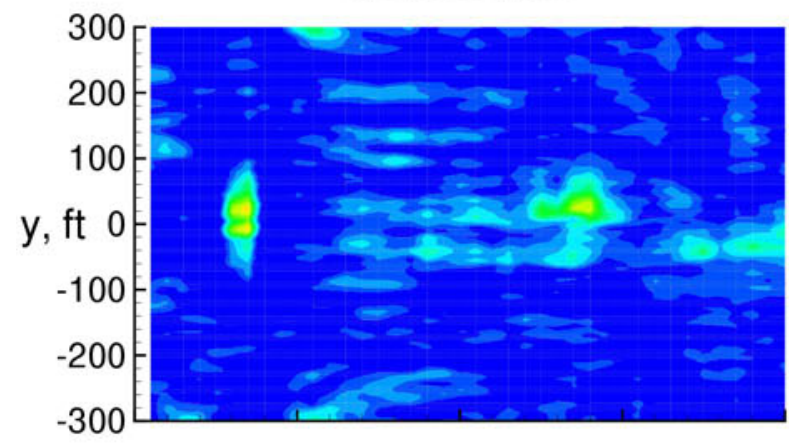

c) $160 \mathrm{~Hz}$ band

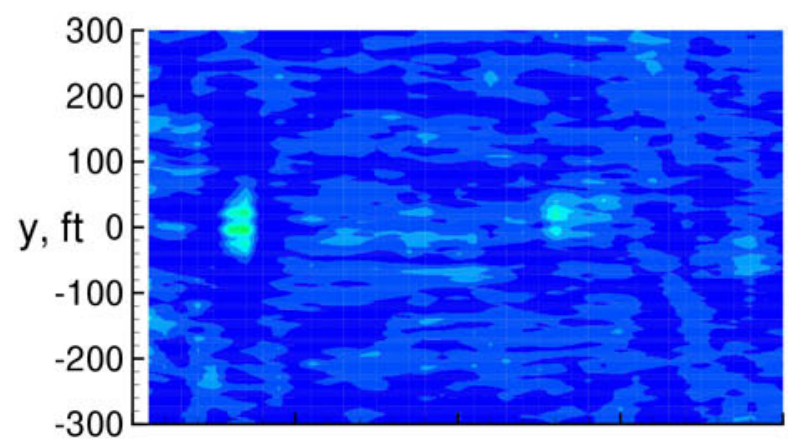

e) $250 \mathrm{~Hz}$ band

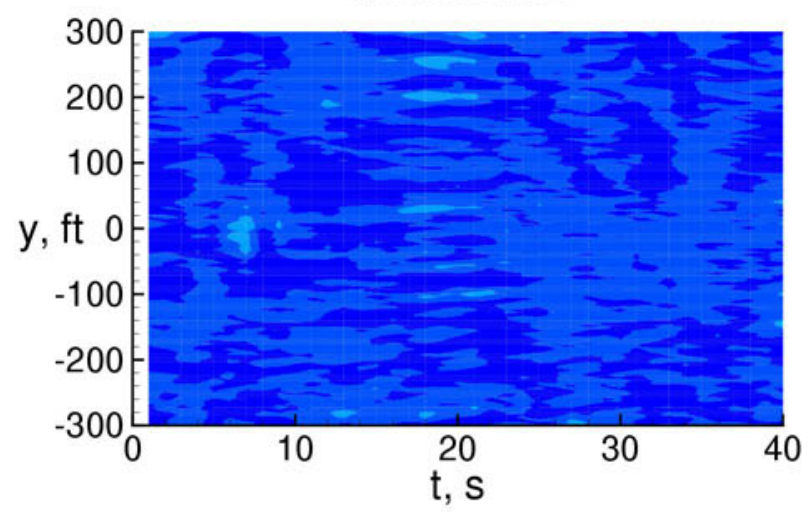

g) $400 \mathrm{~Hz}$ band

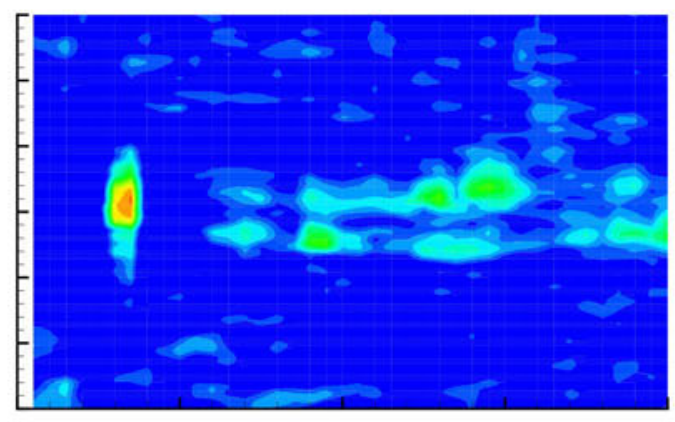

b) $125 \mathrm{~Hz}$ band

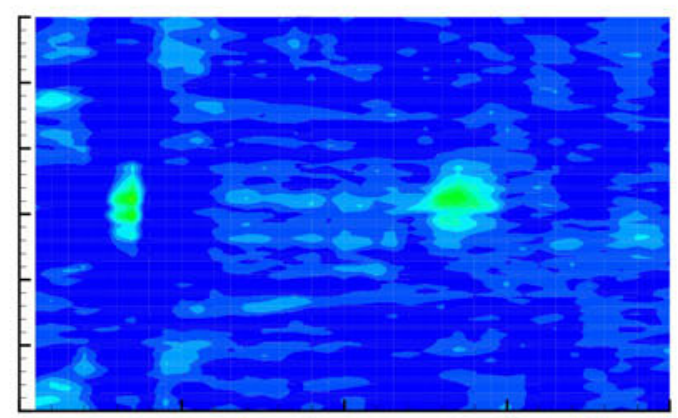

d) $200 \mathrm{~Hz}$ band

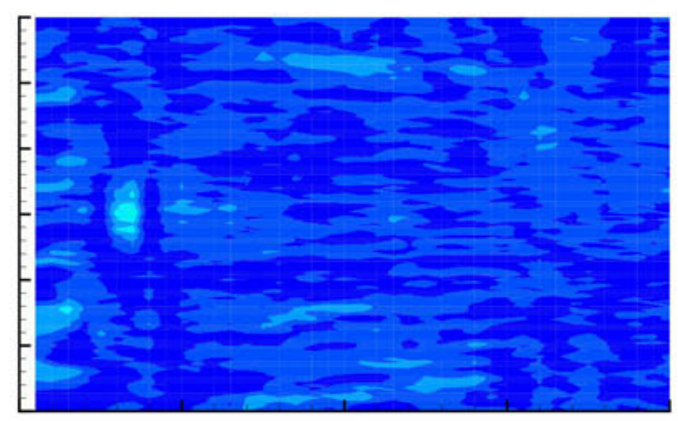

f) $315 \mathrm{~Hz}$ band

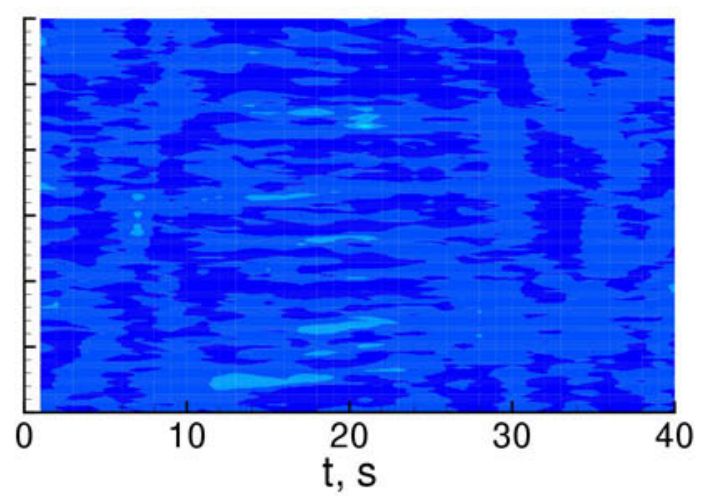

h) $500 \mathrm{~Hz}$ band

Figure 27. Spectral distribution of wake signal from an Airbus A320 (030909_100626). 


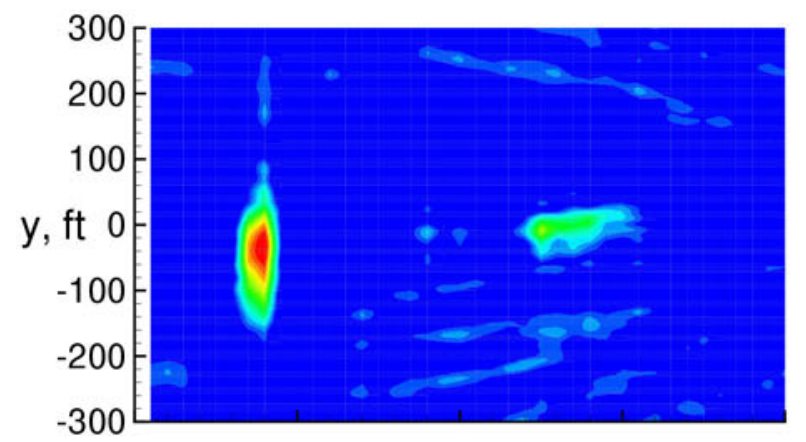

a) $100 \mathrm{~Hz}$ band

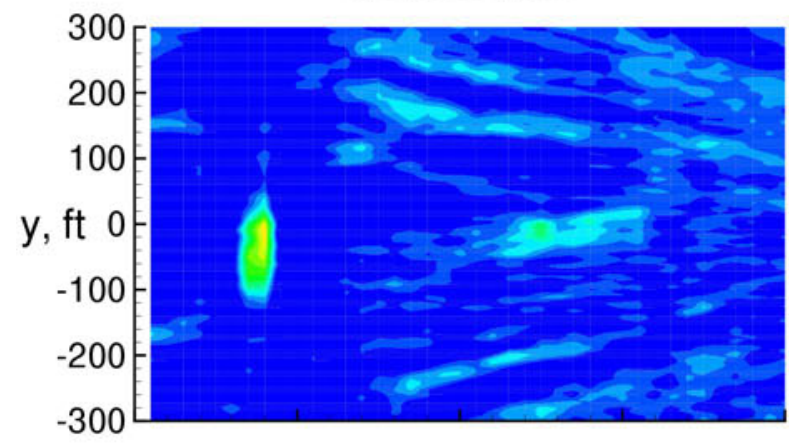

c) $160 \mathrm{~Hz}$ band

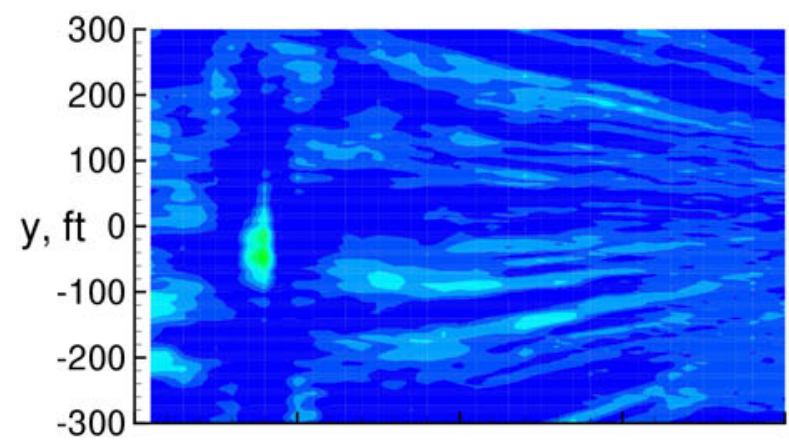

e) $250 \mathrm{~Hz}$ band

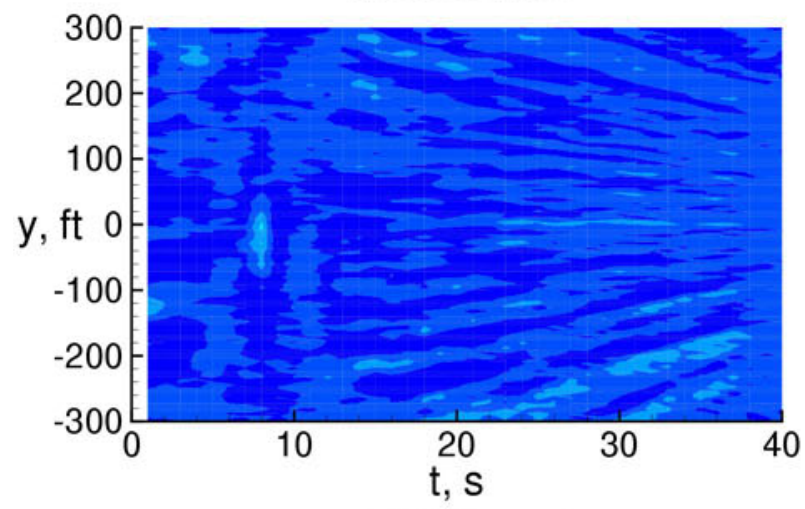

g) $400 \mathrm{~Hz}$ band

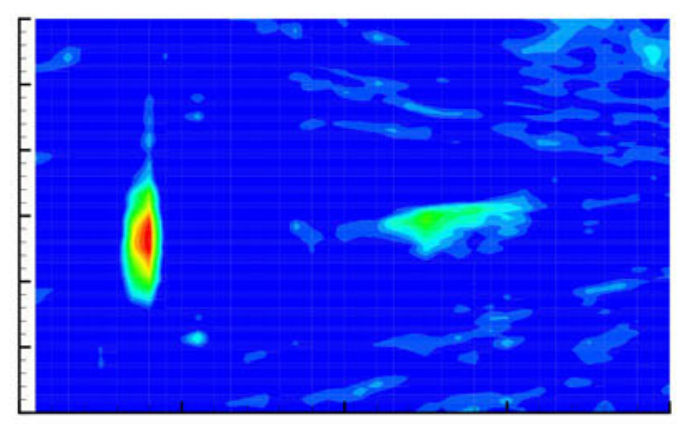

b) $125 \mathrm{~Hz}$ band

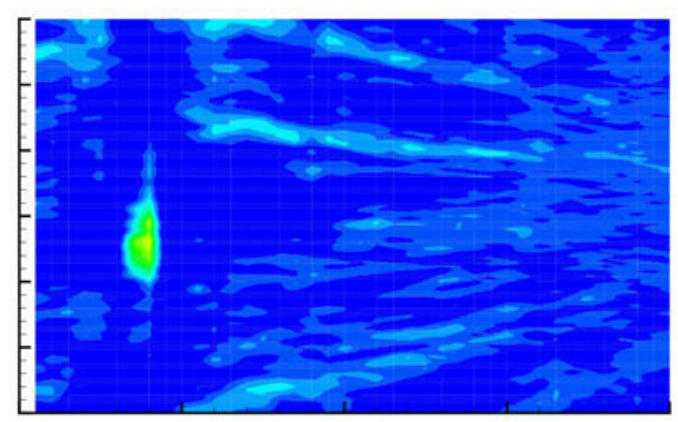

d) $200 \mathrm{~Hz}$ band

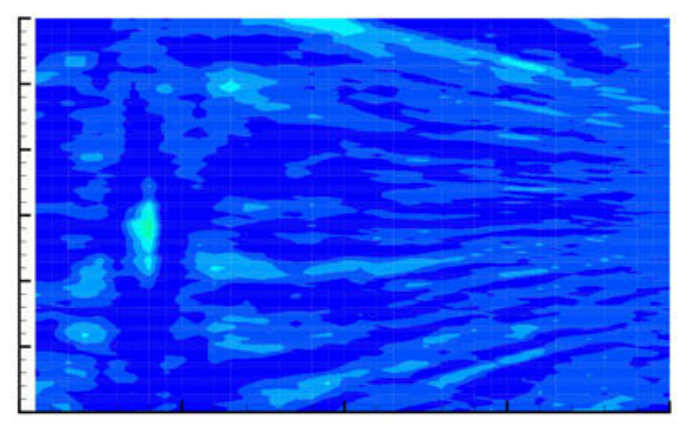

f) $315 \mathrm{~Hz}$ band

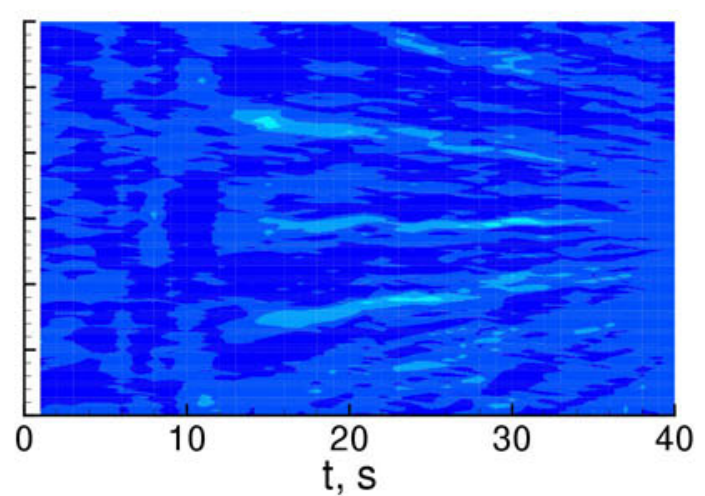

h) $500 \mathrm{~Hz}$ band

Figure 28. Spectral distribution of wake signal from an Airbus A320 (030909_100750). 


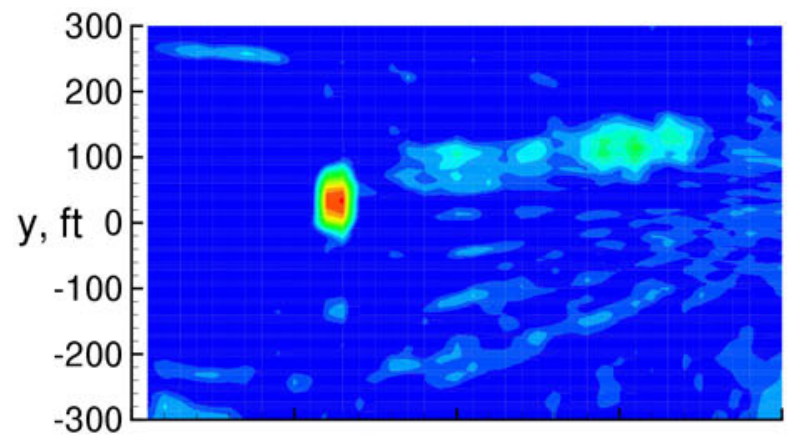

a) $100 \mathrm{~Hz}$ band

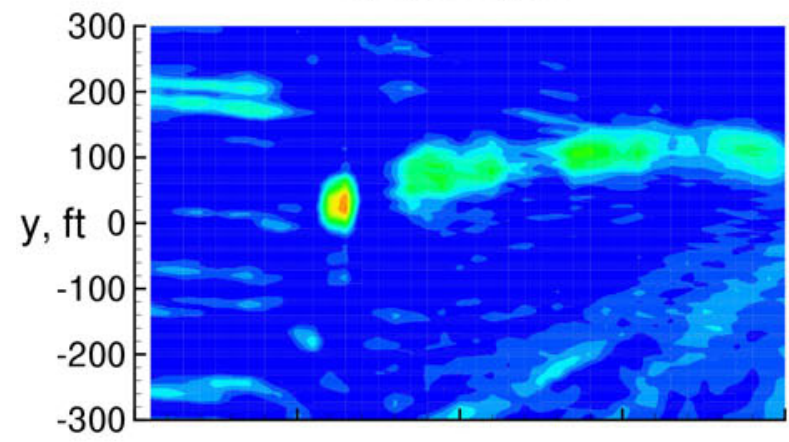

c) $160 \mathrm{~Hz}$ band

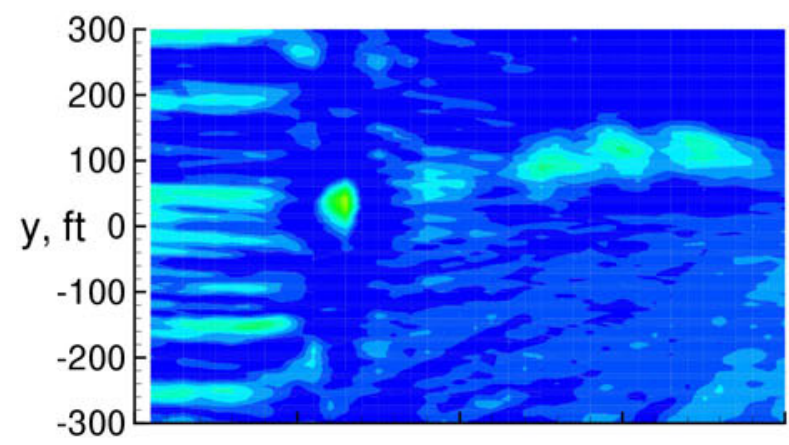

e) $250 \mathrm{~Hz}$ band

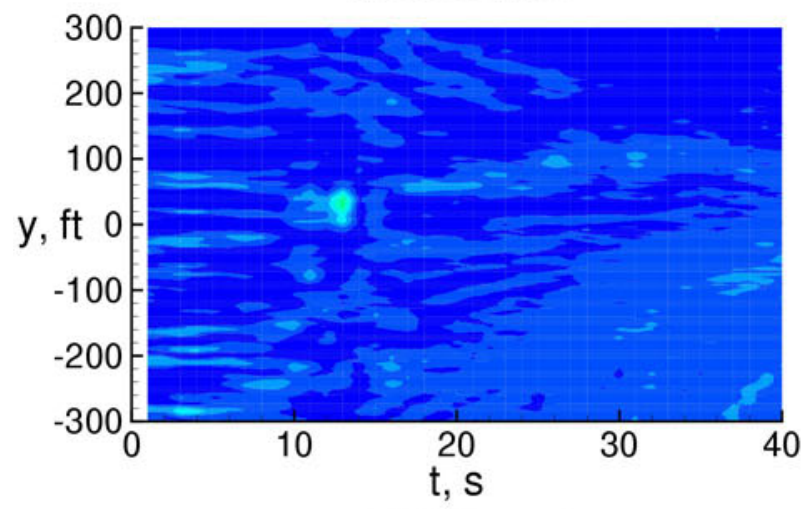

g) $400 \mathrm{~Hz}$ band

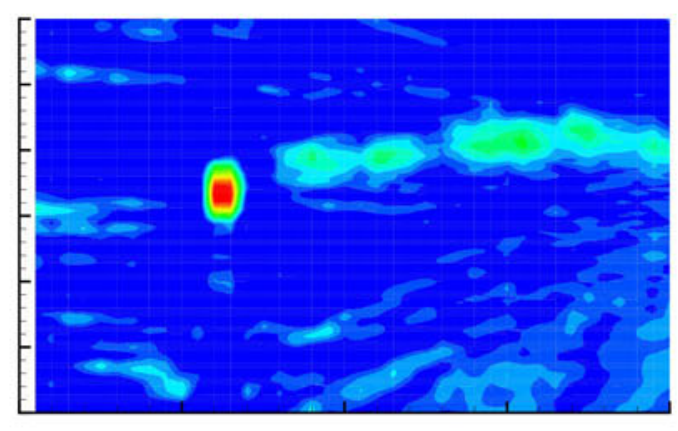

b) $125 \mathrm{~Hz}$ band

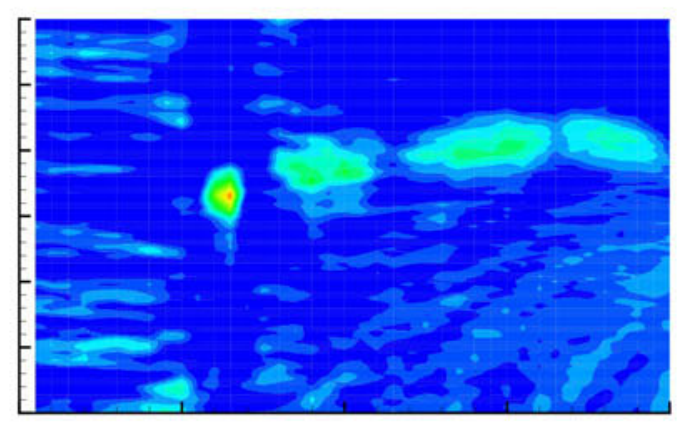

d) $200 \mathrm{~Hz}$ band

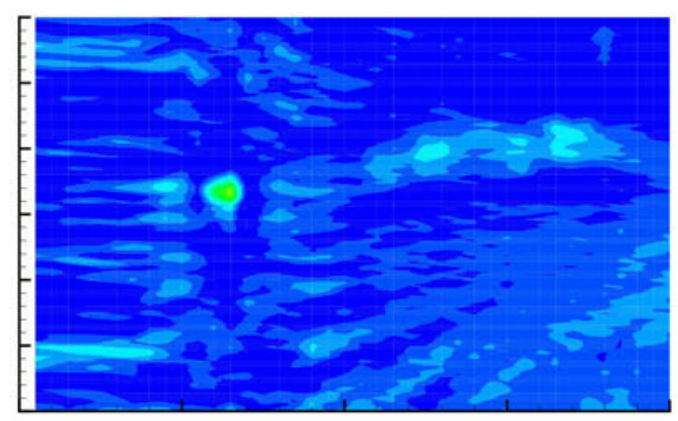

f) $315 \mathrm{~Hz}$ band

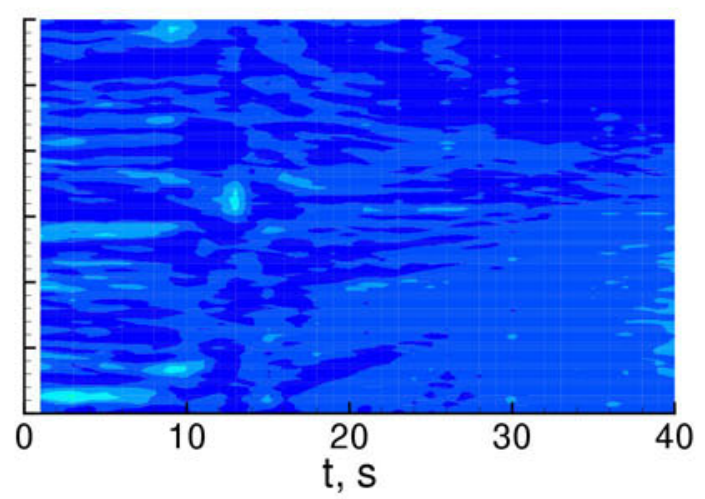

h) $500 \mathrm{~Hz}$ band

Figure 29. Spectral distribution of wake signal from a Bombardier CRJ-200 (030919_084514). 